Humanités numériques

\title{
Du spectacle vivant aux humanités numériques : le projet Dezède
}

From Performing Arts to Digital Humanities: The Dezède Project

Joann Élart et Yannick Simon

\section{(2) OpenEdition}

\section{Journals}

Édition électronique

URL : https://journals.openedition.org/revuehn/408

DOI : $10.4000 /$ revuehn.408

ISSN : 2736-2337

Éditeur

Humanistica

\section{Référence électronique}

Joann Élart et Yannick Simon, « Du spectacle vivant aux humanités numériques : le projet Dezède », Humanités numériques [En ligne], 2 | 2020, mis en ligne le 01 juin 2020, consulté le 16 juillet 2021. URL http://journals.openedition.org/revuehn/408 ; DOI : https://doi.org/10.4000/revuehn.408

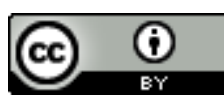

Les contenus de la revue Humanités numériques sont mis à disposition selon les termes de la Licence Creative Commons Attribution 4.0 International. 


\title{
humanités \\ numériques
}

\section{Du spectacle vivant aux humanités} numériques : le projet Dezède

From Performing Arts to Digital Humanities: The Dezède Project

\author{
Joann Élart et Yannick Simon
}

\section{Résumés}

Cet article propose une présentation du site Dezède. Dédié au spectacle vivant, Dezède est une base de données relationnelle réunissant les programmes de plusieurs dizaines de milliers d'événements, pour l'essentiel des opéras, des pièces de théâtre et des concerts. Leur reconstitution normalisée est parfois associée à un appareil documentaire. Dans un premier temps, les auteurs abordent les fondements scientifiques du site et sa place dans le champ de la recherche. Ils s'attachent ensuite à décrire les différentes phases de sa conception avant d'aborder son contenu, ses modes de consultation, le principe des autorités et la communauté de ses utilisateurs et contributeurs.

This article presents the Dezède site. Dedicated to the performing arts, Dezède is a relational database containing the programmes of several tens of thousands of events, mainly operas, plays and concerts. Their standardised reconstruction is sometimes associated with a documentary device. First, the authors discuss the scientific basis of the site and its place in the field of research. They then describe the different phases of its design before addressing its content, its modes of consultation, the principle of authorities and the community of its users and contributors. 


\section{Entrées d'index}

MOTS-CLÉS : arts du spectacle, musicologie, archivage, autorité, base de données, design, enseignement, numérisation, valorisation, Web

KEYWORDS: performing arts, musicology, archiving, authority, database, design, teaching, digitisation, valorisation, Web

Et pourquoi pas Carmen puisqu'il faut bien donner un nom à ce nouveau site consacré au spectacle ? Certes, ce titre emblématique est connu dans le monde entier mais ne risque-t-il pas l'anonymat une fois lancé sur la toile? Et pourquoi pas Boieldieu ? Le clin d'œil aux attaches rouennaises du projet est tentant mais l'orthographe en est trop difficile à mémoriser. Et pourquoi pas Dezède ? C'est finalement à l'issue de sa première présentation publique ${ }^{1}$ que ce projet numérique longtemps sans nom est baptisé.

D-E-Z-E-D-E : trois lettres pour un mot en comportant six, une composition qui interpelle, une construction facile à mémoriser, un nom rare. C'est à Patrick Taïeb, l'un des trois fondateurs du projet, que l'on doit cette trouvaille, car Dezède est bien un patronyme, celui d'une famille de compositeurs, de Nicolas à Florine, du père à la fille. Sans prénom, Dezède est donc un homme ou une femme. Et pourtant, c'est sans nom qu'est né Nicolas, lui qui signait ses partitions « De Z. ", «D. Z. ", "Desaides " ou encore " Dezède ». En 1837, Fétis écrivait qu'" on ignore quelle fut sa patrie " : était-il le fils illégitime d'un prince allemand - de Frédéric II de Prusse, comme on peut le lire parfois - ou était-il né à Lyon? Et quand ? Le mystère reste entier 225 ans après sa mort survenue à Paris en 1792. Fétis rapporte encore qu'à son arrivée à Paris, «il jouissait [...] d'une pension de vingt-cinq mille francs, qui fut doublée à sa majorité. Désirant connaître les auteurs de ses jours, il s'adressa à son notaire ; mais celui-ci le prévint que ses démarches seraient inutiles, et qu'en les continuant il s'exposerait à perdre son revenu. Il ne tint pas compte de cet avis, continua ses recherches, ne découvrit rien, et fut privé de sa pension. Ce fut alors qu'il songea à tirer parti de ses talents pour assurer son existence " (Fétis 1837, 302). Mais pour les dix-huitiémistes, Nicolas Dezède est loin d'être un inconnu ; il est une célébrité de son temps qui marqua pas moins de trois générations d'amateurs de théâtre avec des œuvres comme Les Trois Fermiers (1777), Blaise et Babet (1783), Alexis et Justine (1785) ou Auguste et Théodore (1789). Ces pièces figurent parmi les plus grands succès du répertoire de l'Opéra-Comique et de la Comédie-Française entre la fin de l'Ancien Régime et la Restauration. Véritable énigme en soi, ce nom justifiait presque à lui seul le sens de notre projet numérique, qui pourrait permettre, à défaut de la résoudre, de reconstituer la carrière du compositeur qui l'a porté.

Inspiré par des études anciennes dont les grandes lignes seront rappelées dans un premier temps ("L'archéologie du spectacle »), Dezède ${ }^{2}$ puise ses ressources méthodologiques dans des programmes de recherche portant sur l'histoire du concert en France à partir desquels, tout en l'étendant au théâtre au sens large du terme, le projet s'est structuré autour d'un principe : celui de reconstituer des chronologies de 
spectacle, avec une approche renouvelée et adossée à un outil innovant (Élart, Simon, et Taïeb 2012). Dans un deuxième temps ("Concevoir et créer un nouvel outil numérique »), nous reviendrons sur les étapes qui ont permis de bâtir ce projet entièrement conçu avec des technologies open source à partir de 2011. Le site accueille des corpus variés, établissant une histoire du spectacle vivant in progress, sous toutes ses formes, dans un espace géographique sans frontières et dans un espace temporel sans limites. Dezède se démarque ainsi des "outils fermés ", à l'image des bases de données que développent notamment les institutions pour décrire exclusivement leur propre répertoire ${ }^{3}$. Les contenus et les modes d'interrogation feront l'objet de la troisième partie ( Les contenus et les modes de recherche ») : ce sont des événements qui, mis bout à bout, composent un corpus éclectique et par nature infini du spectacle vivant ou qui, lorsqu'ils sont classés dans des dossiers, apportent un éclairage original sur l'un des aspects de cette histoire. Dans tous les cas, les événements sont validés par les sources ayant permis de les reconstituer. La méthode historique, plus qu'une inspiration, est ici fondatrice de la pensée générale de Dezède. En son cœur, des milliers d'autorités sont autant d'unités d'information que gère une base de données mettant en relation une matière dense et complexe, comme nous le présenterons dans un quatrième temps ("Au cœur du système : les autorités »). Projet de recherche collaboratif et contributif, Dezède est mis gratuitement à la disposition d'une communauté composée de chercheurs, d'institutions, mais également d'étudiants (cinquième point, " La communauté Dezède »). Outil de recherche et de valorisation, Dezède n'en est pas moins un outil pensé pour l'enseignement universitaire, comme nous le verrons dans un dernier temps (« L'enseignement »).

\section{L’archéologie du spectacle}

S'inscrivant dans un périmètre scientifique très large à la croisée de plusieurs disciplines en sciences humaines, Dezède a pour objet d'étude principal le spectacle vivant, sans limites chronologiques et géographiques. La conception du programme a néanmoins reposé sur les outils traditionnels que les fondateurs manipulaient dans leur propre champ de recherche. Ils sont par conséquent à l'origine de leurs réflexions et du modèle numérique auquel ils sont parvenus. Somme toute, les chronologies de spectacles lyriques et de concerts publiées étaient suffisamment peu nombreuses pour en permettre une analyse générale. De fait, si certains théâtres lyriques et certaines sociétés de concerts tiennent depuis longtemps le journal de leurs activités artistiques, c'est davantage pour des raisons internes et administratives que par souci de mettre à la disposition des chercheurs et du grand public le recensement exhaustif de leurs activités. De plus, ces catalogues n'ont pas tous eu le destin des journaux de bord de l'Opéra et de l'Opéra-Comique, fort bien conservés, alors que d'autres, victimes d'une politique d'archivage erratique, ont disparu. Dans ces conditions, la reconstitution des activités des théâtres lyriques et des sociétés de concerts à partir de sources variées s'apparente à une forme d'archéologie du spectacle, et devient un outil fondamental pour l'étude de la réception, au sens large du terme. 
Dans le domaine du théâtre, des travaux pionniers produits au $\mathrm{XIX}^{\mathrm{e}}$ siècle visent à écrire l'histoire des théâtres de France à partir des archives et de la presse : ce sont les études de Jules-Édouard Bouteiller $\left(1860^{4}\right)$ ou de Charles-Pierre Vauclin (1912) sur Rouen, celles d'Arnaud Detcheverry sur Bordeaux (1860), d'Adolphe Gilles sur Montpellier (1908) [neuf registres et dix cahiers de 1790 à 1908], de Léon Lefebvre sur Lille (1901), de Léandre Moreau sur Marseille (1872) ou encore de Charles Vesque sur Le Havre (1875). Ces histoires reconstituent partiellement une série d'événements choisis pour leur importance dans la vie des théâtres de province - premières représentations des œuvres, faits marquants dans l'histoire locale comme la représentation d'un artiste de renom ou la visite d'une personnalité politique de premier rang, anecdotes, etc. - selon une méthode de traitement chronologique.

Il est néanmoins très probable que les deux ouvrages qu'Antoine Elwart consacre à des sociétés de concerts en 1860 et 1864 contiennent les premiers exemples de chronologies systématiques publiées en France. La première concerne la Société des concerts du Conservatoire (Paris) entre 1828 , l'année de sa fondation, et 1859 (Elwart $1860^{5}$ ). La seconde se limite aux trois premières saisons des Concerts populaires de musique classique que dirige Jules Pasdeloup entre 1861 et 1863 à Paris (Elwart $1864 \mathrm{~b}^{6}$ ). Dans les deux cas, l'auteur transcrit les programmes de salle dont il reprend la présentation (figure 1) : les titres y précèdent le nom du compositeur ; les intitulés ou les indications de tempo des mouvements des œuvres en plusieurs parties, comme les symphonies, sont détaillés ; de même, les noms des solistes ; seuls les titres des opéras sont en italique ; à l'occasion, les dates du compositeur sont mentionnées. Chaque liste, dont les occurrences sont numérotées, est surmontée de l'indication du rang du concert dans la série, de la date et de l'heure de l'événement. Des appels de note renvoient fréquemment à des commentaires sur l'œuvre, l’interprétation ou sa réception.

FIGURE 1. SIXIÈME CONCERT POPULAIRE, DONNÉ LE 29 NOVEMBRE 1863

\section{Ge CORCBRT.}

Le Dimanche 99 novembre 1863 , à deux heures précises.

1. Ouverture d'Il Matrimonio segreto, de Cimarosa.

2. Symphonie en la mineur, de Mendelssohn (1).

Introduction, Allegro agitato, Scherzo, Adagio, Allegro vivace.

3. Rigodon de Rameau, né à Dijon en 1683, mort en 1764.

4. Adagio du septuor de Beethoven, exécuté par MM. Auroux (clarinette), Espeignet (basson), Schlottmann (cor), et tous les instruments à cordes.

5. Ouverture de Preciosa, de Weber. 
Outils de recherche précieux et globalement fiables, les deux répertoires d'Elwart peuvent se voir reprocher de ne pas mentionner l'origine des sources, de ne pas permettre l'identification des œuvres, de ne pas uniformiser la présentation des titres et d'être dépourvus d'index. Sans équivalent jusqu'à la fin du $\mathrm{XIX}^{\mathrm{e}}$ siècle, ils apparaissent dans une période qui semble marquée par un besoin croissant de recenser les programmes des concerts et d'établir des rétrospectives. En témoignent notamment les livres de Paul-Marie de Toyon, intitulés La Musique en 1864 (Toyon 1865) et La Musique en 1865 (Toyon 1866), ceux de Camille Bellaigue (L’Année musicale), couvrant les années 1886 à 1893 (Bellaigue 1888), ou encore ceux d'Oscar Comettant, spécifiquement dédiés aux salons de la maison Pleyel entre 1893 et 1898 (Comettant 1894).

Il est intéressant de constater qu'un siècle plus tard, un travail universitaire de grande ampleur ne se distingue guère de la méthode appliquée par Elwart au recensement des programmes de concerts. Tel est bien le cas de la thèse de troisième cycle qu'Élisabeth Bernard soutient en 1976. Concert symphonique à Paris entre 1861 et 1914 propose, après un premier volume consacré à leur histoire, trois autres tomes réunissant les programmes des concerts des principales sociétés de concerts parisiennes (Bernard $1976^{7}$ ). En outre, dans deux autres volumes indépendants de ce premier ensemble et probablement postérieurs, Élisabeth Bernard recense les Programmes de la Société des concerts du Conservatoire de 1828 à 1870 (Bernard 1980). Fondamentalement, la méthode appliquée diffère peu de celle d'Elwart même si le nom du chef d'orchestre est mentionné et que chaque événement est numéroté pour permettre l'indexation des individus et des œuvres (figure 2). On remarquera aussi que les noms figurent désormais avant les titres. Toutes ces informations restent simplement transcrites sans tentative de normalisation.

FIGURE 2. CONCERT DU 11 FÉVRIER 1866

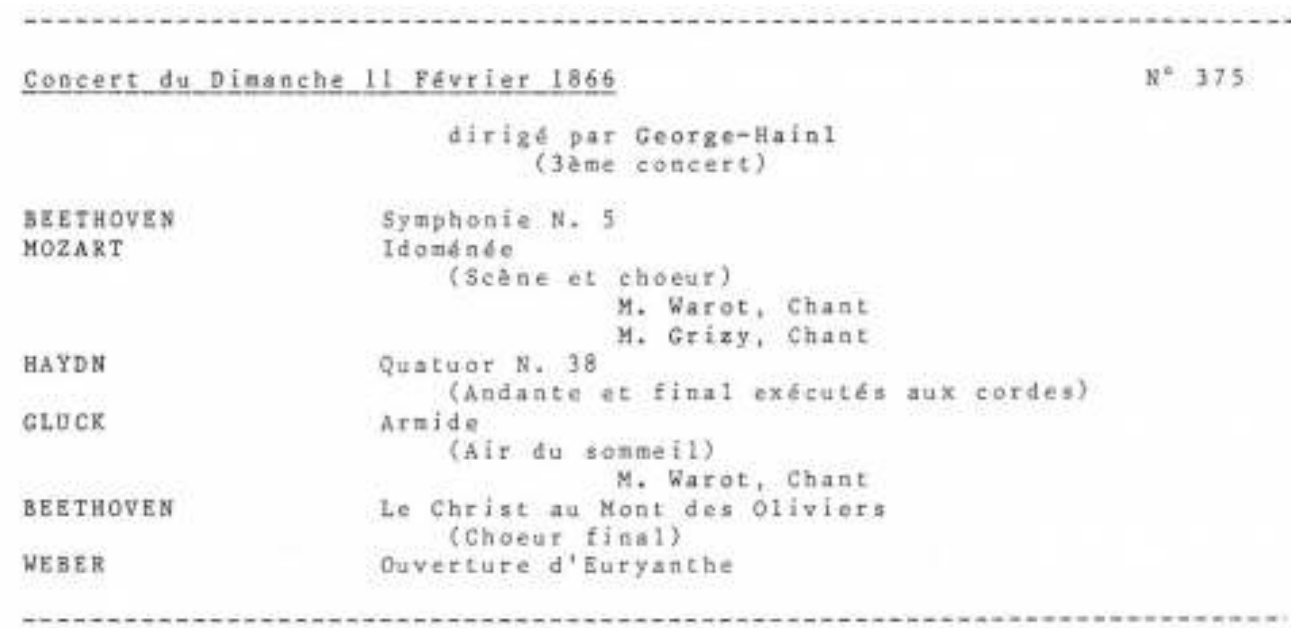

Extrait des Programmes de la Société des concerts du Conservatoire d'Élisabeth Bernard (p. 128)

Bien que rédigé à la fin $d u \mathrm{xIx}^{\mathrm{e}}$ siècle, le texte de Constant Pierre consacré au Concert spirituel n'est publié pour la première fois qu'en 1975 (Pierre 1975, 2000). C'est par conséquent de cette période que date la mise en forme du répertoire qui se distingue de celle des modèles précédents $^{8}$. Le répertoire des programmes du Concert spirituel entre 1725 et 1790 contient de nombreuses abréviations, un numéro pour chaque concert, la mention de la source dans laquelle le programme a été copié 
et un index facilitant l'accès au corpus (figure 3). Les éléments du programme matérialisés par le titre des œuvres sont enchaînés dans le même paragraphe, et non plus superposés grâce un retour à la ligne après chaque entrée. Ces titres sont transcrits en italique, mais toujours avant le nom du compositeur, et présentés dans une forme modernisée sans références aux catalogues des compositeurs. Le document ne contient aucun appel de note, mais peut préciser tout un ensemble de caractéristiques signalant ici la première apparition d'un artiste dans l'institution (" $1^{\text {re }}$ fois »), là, la première exécution d'une œuvre («nouv. »).

FIGURE 3. CONCERT DU 18 JUIN 1778 AU CONCERT SPIRITUEL, AU COURS DUQUEL A ÉTÉ CRÉÉE LA SYMPHONIE $N^{O} 31$ DE MOZART

983. I8 juin (Fette-Dieu.) (JP. des 16, p, 665 et 18, p. $674 ;$ A. du 18, p. 926.) Nouv. symph., Amadeo Mozart. - Air ital., Jomelli ch, Mme Saint-Huberti. - Conc. bon ex. Destouches ( $\mathrm{I}^{\mathrm{re}}$ fois.) - M.2v, Gossec ch. Chéron et Legros, - Son vcelle, ex. [L. A.] Jannson. - Conc. von ex. Mile Deschamps. - Non so d'onde viene, [J. C.] Bach ch. Raaf. - Air ital. redemandé, Gluck ch. Mme Saint-Huberti. - Susanne, nouv, orat,, Bambini : Mile Malpied, Ire fois, Moreau, Chéron Péré, Deschet.

Extrait de l'Histoire du Concert spirituel de Constant Pierre (p. 309).

Nous renvoyons à l'événement reconstitué dans Dezède à titre de comparaison : https://dezede.org/evenements/id/46924/.

Plus tardif et consacré au théâtre lyrique, le répertoire du Théâtre-Italien publié par Jean Mongrédien au terme de vingt années de travail occupe une place singulière parmi les modèles de Dezède (Mongrédien 2008) (figure $4^{9}$ ). Publié en 2008, il est le résultat d'un projet collaboratif ayant compté en son sein plusieurs chercheurs à l'origine de Dezède. Les huit volumes de cette vaste entreprise contiennent, d'une part, la chronologie du Théâtre-Italien à Paris de 1801 à 1830, d'autre part, les annonces et les comptes rendus parus dans la presse. Les titres des ouvrages lyriques y sont normalisés suivant une méthode inspirée des pratiques du catalogage ${ }^{10}$. Les programmes de spectacle ne sont plus recopiés mais reconstitués sur la base de sources dont la transcription est fournie conjointement suivant un modèle unique. Le tout est présenté sous la forme d'une chronologie hybride, mélangeant, d'un côté, le relevé des représentations de l'institution et, de l'autre, les annonces de presse classées au jour de leur publication sans être précisément associées à la représentation qu'elles décrivent. Ce dernier point est une différence de conception marquante avec la structure de Dezède, dans lequel les sources sont toujours associées à un événement.

FIGURE 4. DÉBut DE LA NOTICE DU 13 SEPTEMBRE 1806

\title{
SAMEDI 13 SEPTEMBRE 1806
}

\section{Représentation du jour}

\author{
La Prova d'un opera seria, dramma gio- \\ coso per musica en deux actes (F. Gnecco, \\ compositeur et librettiste) \\ MM. Barilli, Bianchi, Carmanini, Zardi, Lupi \\ $\mathrm{M}^{\text {mes }}$ Canavassi, Salucci [171]
}

\section{Échos et nouvelles}


" м1 Lataianı, retenue a Paris jusquau milieu de la semaine prochaine, s'est rendue aux instances des personnes qui n'ont pu trouver place à son dernier concert et au voeu général du public. En conséquence on aura le plaisir de l'entendre encore une fois la veille de son départ. Ce concert est fixé au mercredi 17 du courant. 》 [200]

\section{Comptes rendus}

«Opera-Buffa.

«La Prova d'un opera seria (La Répétition d'un grand opéra) attire en ce moment la foule au théâtre Louvois. La musique de Gnecco est charmante et parfaitement exécutée par M ${ }^{\text {me }}$ Canavassi, MM. Barilli et Carmanini. Nous ne parlerons point du plan de la pièce : on sait ce que sont les poèmes italiens. 》 [231], année $1806, t . V$, p. 173.

«Variétés. Littérature. Sciences. Arts.

«[...] Rien de nouveau cette semaine sur les trois grands théâtres. Un nouvel opéra bouffon, intitulé La Prova d'un opera seria (La Répétition d'un opéra sérieux) a obtenu sur le théâtre de l'Impératrice le succès le plus brillant et le mieux mérité. L'auteur de la musique, le seul dont il puisse être question quand il s'agit dopéras italiens, est il signor Gnecco, dont personne n'avait entendu parler en France avant ce début brillant. À la vérité, les morceaux des autres compositeurs que l'on a ajoutés à la musique un peu faible de cet opéra n'ont pas peu contribué à en assurer le succès. Parmi ces morceaux, on distingue la polonaise de Della Maria, La gioia, la calma. Ces petits airs sont toujours vivement applaudis, quoiqu'ils se ressemblent tous. Quand on a entendu la polonaise de Viotti, on les a toutes entendues. Lair de Trento Tremo da capo al piè, etc. est fort beau et très bien chanté par $\mathrm{M}^{\mathrm{me}}$ Canavassi. On fait toujours répéter le trio dans le genre sérieux, soutenu par un chceur, Le nostre lagrime ; le chant en est admirable et il est parfaitement exécuté par $\mathrm{M}^{\text {mes }}$ Canavassi et Salucci et par M. Bianchi qui, dans la pièce, fait le rôle de l'Amant de la Prima donna et dans le trio, celui de Zamore a Trebizonda. Il chante avec une grande expression le récitatif qui précède ce beau trio : 

taire tendant à l'exhaustivité, le répertoire de J. Mongrédien est un objet unique dans la musicologie française. Il est le résultat d'un projet éditorial sans commune mesure qui témoigne néanmoins d'un passé révolu depuis l'émergence du numérique.

\section{Concevoir et créer un nouvel outil numérique}

\section{Aux origines du projet}

Dezède puise ses origines dans un programme de recherche intitulé "Répertoire des programmes de concerts en France " (RPCF) et dirigé par deux anciens étudiants de Jean Mongrédien : P. Taïeb et Hervé Lacombe ${ }^{11}$. Constitué autour du détachement de P. Taïeb à l'Institut universitaire de France entre 2000 et 2005, puis autour d'un projet financé par l'Agence nationale de la recherche entre 2006 et 2009, ce programme est partagé par plusieurs dizaines de chercheurs du monde entier pendant une décennie. Dezède s’inspire plus particulièrement du " mode d'emploi " (Élart et al. 2005) élaboré au sein des séminaires pour unifier et uniformiser le travail de tous les participants. Une base de données " locale " développée à partir des principes établis par la méthode devait par ailleurs préparer la publication des répertoires. Bien que satisfaisante, cette solution provisoire est devenue rapidement ingérable : non seulement elle supposait l'acquisition par tous les membres du groupe une cinquantaine de chercheurs - de licences commerciales, mais elle obligeait à les former à distance, par téléphone ou par courriel, puisque la base n'était pas partagée sur le Web ; autres problèmes jamais résolus, ceux des capacités limitées du logiciel et de l'hébergement des données. Si les efforts menés pour concevoir un outil informatique n'ont pas abouti à cette époque, les expériences acquises ont permis toutefois d'établir un cahier des charges pour le réaliser : la solution attendue devait être pérenne, gratuite, sécurisée, souple, illimitée, contributive et dématérialisée ; en outre, elle devait proposer une centralisation des données pour permettre leur croisement et leur valorisation. C'est sur cette base, à laquelle est venu s'ajouter le souhait d'élargir la méthode à l'ensemble des spectacles, que le projet est lancé en octobre 2011, au moment où s'opèrent les premiers contacts avec Bertrand Bordage, le concepteur du programme informatique, activement impliqué dans les communautés de l'open source et fondateur de de l'agence de conception de sites NoriPyt.

Après quatre mois de travail avec le développeur, le prototype de $D e$ zède permet déjà de produire une chronologie. Lors de la présentation inaugurale le 23 février $2012^{12}$ est créé en direct le premier événement de Dezède, une soirée hybride donnée au Théâtre des Arts de Rouen le 15 janvier 1828 à l'occasion de l'anniversaire de la naissance de Molière ${ }^{13}$. Toujours en phase d'incubation, la version alpha est mise en ligne le 28 septembre 2012 et la version bêta propulsée le 21 novembre 2012 dans le cadre d'une seconde journée d'étude ${ }^{14}$. Le choix de mettre en ligne le prototype de Dezède comportait certes des risques, mais nous souhai- 
tions, avec tous les défauts d'un projet en construction, passer à une première phase de prise en main du logiciel en confiant l'outil à quelques chercheurs et à quelques groupes d'étudiants. Riche d'enseignements, cette première expérience de terrain a permis, d'un côté, d'améliorer et de corriger le programme et, de l'autre, de produire plusieurs milliers de données dans la base. La première "version définitive " est inaugurée le 21 février 2013 dans le cadre d'un colloque organisé à l'Opéra-Comique (Élart et al. 2013), puis la "version 2.0 ", à l'adresse définitive https://dezede.org, le 21 mai 2014 dans le cadre d'un nouveau colloque à l'OpéraComique (Élart et al. 2014). À cette occasion, la nouvelle interface du site - toujours en fonction - est dévoilée. Dezède est ensuite présentée à différentes occasions : en 2013, au colloque This Is The Modern World (Élart 2013a), au Network Meeting du groupe Francophone Music Criticism (Élart et Simon 2013a) et à la journée professionnelle de l'Association internationale des bibliothèques musicales (Élart et Simon 2013b) ; en 2014, à l'Institut de recherche sur la Renaissance, l'âge classique et les Lumières (UMR 5186) (Élart 2014a) ; en 2015, à l'occasion de deux tables rondes du colloque Remettre en jeu le passé. Métamorphoses du corpus des Registres de la Comédie-Française ${ }^{15}$; en 2016, au séminaire de l'IMEC (Élart 2016a) et à la journée du consortium Musica ${ }^{16}$; en 2017, au workshop Music Performance Ephemera (Simon 2017b).

Parallèlement à sa conception et à sa réalisation, l'outil numérique adopte une forme légale, institutionnelle et éditoriale. Un accord de copropriété réunit en 2014 les trois enseignants-chercheurs à l'origine du projet $^{17}$, leurs laboratoires - le GRHis (EA 3831), le CÉRÉdI (EA 3229) et l'IRCL (UMR 5186) - et leurs universités de rattachement - Rouen Normandie et Paul-Valéry-Montpellier 3. Sa signature est précédée par un accord de cession des droits patrimoniaux des inventeurs au profit de leurs institutions. Accueilli sur les serveurs de la TGIR Huma-Num en juin 2013, Dezède est référencé en tant que ressource numérique open source avec le numéro d'ISSN 2269-9473 en octobre 2013 et en tant que marque déposée à l'INPI en décembre 2013. Un comité éditorial rassemblant les trois inventeurs est mis en place pour évaluer les projets et accompagner leurs porteurs. 


\section{Technologies, design et navigation}

D'un point de vue technologique, la conception de Dezède repose sur quatre idées fondatrices qui ont constitué les points forts du cahier des charges pour le recrutement du développeur : ouverture, pérennité, stabilité et ergonomie. L'expérience de la recherche collective au sein du groupe RPCF a déterminé le premier point, car nous avions mesuré l'inadéquation entre la volonté de partager un outil commun et l'obligation pour tous les membres de s'affranchir des licences commerciales et des mises à jour d'un logiciel de base de données. Il s'agissait donc, dès l'origine en 2010, de développer un outil ayant recours exclusivement à des solutions fondées sur le code source ouvert. Observant les principes de l'open source, notre code est de fait accessible depuis décembre 2011 sur un dépôt GitHub. Ce choix fondateur a déterminé celui des solutions techniques, que nous souhaitions les plus pérennes. C'est pourquoi nous avons suivi les conseils de notre développeur, en optant pour le langage de programmation Python, qui permet d'écrire du code extrêmement lisible et qui simplifie les opérations de maintenance. Outre le langage de programmation, les principales solutions techniques utilisées pour le développement de Dezède sont Django pour la boîte à outils Web, PostgreSQL et Redis pour la base de données, Elasticsearch pour le moteur de recherche, Bootstrap et Less pour le design. Par ailleurs, la mise en place d'une suite de tests automatiques couvrant la majeure partie des fonctionnalités de Dezède garantit une grande stabilité au programme, et force est de constater que, depuis sa mise en service, les bogues sont en effet très rares. Enfin, l'ergonomie a guidé le développement et la modélisation de Dezède, d'un bout à l'autre du logiciel, du back office au front office, du contributeur au lecteur. Que l'utilisation de cet outil soit la plus intuitive possible, tel est notre vœu ; que l'outil suggère de lui-même les modes d'utilisation, d'interrogation ou de contribution, sans intervention humaine, telle est notre quête numérique.

C'est également cette sensibilité et cette approche du numérique qui a guidé la réalisation du design. L'application de front office devait être simple et intuitive, présenter clairement l'outil, les contenus et les contributeurs. La connexion au site passe par une page d'accueil réduite, autour du logotype et des menus en en-tête, à un carrousel de dossiers mis en avant en fonction de l'actualité culturelle et de la production des utilisateurs, à l'affichage des partenaires qui bénéficient d'un accès direct à leurs données, et à un lien vers le Carnet Dezède (https://dezede.hypotheses.org) et la page Facebook (https://www.facebook.com/ dezede.org). Cette page facilite par ailleurs les démarches qui engagent librement toute personne désireuse d'enrichir la base de données - le bandeau "contribuer " donne accès à un formulaire de soumission de projet qui est étudié par le comité éditorial. Dans le même souci de clarté, les menus sont réduits au nombre de trois : une première entrée par les « dossiers »- qui propose une sélection d'événements décrivant une thématique donnée -, une deuxième par les "événements » - qui permet une recherche simple ou avancée dans tous les contenus visibles - et une troisième par la «bibliothèque " - qui regroupe un ensemble d'études sur le spectacle. Le moteur de recherche articule ces " menus de contenus » (dossiers, événements, bibliothèque) avec des «menus techniques » (in- 
dex général, équipe, partenaires, informations générales, bibliographie, menu multilingue ${ }^{18}$, accès à l'administration, API, sources sur GitHub). Tous ces menus disposés en en-tête sont accessibles dans les différentes pages du site.

La charte graphique est quant à elle volontairement " rétro ", car il s'agit, par des couleurs privilégiant les tonalités douces et écrues, ou encore par une police avec empattements et ligatures respectant la typographie traditionnelle - les apostrophes sont par exemple typographiques et non dactylographiques (courbes et non droites) ${ }^{19}-$, de rechercher un confort de lecture et une lisibilité à la fois adaptée au Web mais rappelant également ceux du livre. Cette charte graphique n'en est pas moins souple et flexible, puisqu'elle est responsive et s'adapte à tous les supports (ordinateurs, tablettes, smartphones).

Le logotype Dezède est inspiré d'un manuscrit appartenant au fonds du Théâtre des Arts conservé à la bibliothèque municipale de Rouen, qui reproduit la mention d'auteur figurant sur la page de titre d'Auguste et Théodore (cote [Théâtre 52] ${ }^{20}$. Il s'agissait ainsi de rendre hommage à cette bibliothèque théâtrale qui a joué un rôle fondateur dans l'émergence du projet Dezède ${ }^{21}$. Il s'agissait également de souligner le rapport qu'entretiennent les données produites avec les sources traitées pour leur reconstitution : Dezède est fondé sur la méthode d'investigation historique, au cœur de laquelle la source est reine. Et quoi de plus marquant pour l'esprit que d'associer l'idée d'enquête historique à un manuscrit ? Précisons toutefois que cette reproduction n'en est pas une puisqu'il s'agit d'une réalisation en dessin vectoriel inspirée de cette mention par Bertrand Bordage. Enfin, de ce logotype, il a été tiré un favicon, qui peut être utilisé en raccourci dans les menus de navigation et qui a été intégré à gauche de la barre de menus en en-tête ; c'est d'ailleurs par lui que l'utilisateur peut revenir à la page d'accueil à tout moment.

\section{Les contenus et les modes de recherche : les événements, les dossiers et leurs sources}

\section{Les événements}

L'événement est l'unité de base et de mesure de Dezède. Qu'il s'agisse de la représentation d'une pièce de théâtre ou d'un opéra, d'un concert ou de tout autre forme de spectacle, public ou privé, dans un lieu fermé ou ouvert, avec ou sans interprète, le rassemblement d'un groupe d'individus - le public - assistant à un spectacle constitue un événement. Le spectacle vivant en est le domaine de prédilection, même si dans l'absolu aucune forme ne présente un caractère d'incompatibilité avec le site (projections cinématographiques, expositions, manifestations sportives, etc.) La construction des événements s'appuie sur des éléments d'identification classiques, comme nous pouvons le voir dans l'exemple ci-dessous (figure 5) : date, lieu, horaire, distribution, programme, notes éventuelles, source(s). 


\section{Dimanche 8 mars 1863}

Paris, Cirque d'hiver, 14 h00

Pasdeloup (J.) [chef d'orchestre], Orchestre des Concerts Pasdeloup

\section{Programme}

1. Hardn (J.) [comp.], Symphonie n' 93, en ré majeur Hob. 193

2. Mendelssohn Bartholor (F.) [comp.], Symphonie pour chour, soliste et orchestre $n^{\prime \prime} 2$, ib. Allegretto un poco agitato

3. Mozırt (W. A.) [comp.], Sonate pour piano en la majeur, 3. Alla Turca [Marche turque], Allegretto [orchestré par $\mathrm{Pr}$. Pascal]

4. Beethoven (L. van) [comp.], Symphonie $n^{*} 4$, en si bémol majeur op, 60

5. RossıNI (G.) [comp.], Séminamis, Ouverture

\section{Notes}

\section{4e de la je série}

1. En contradiction avec l'annonce, Elwart (Histoire des Concerts populaires de musique classique, p. 67) et Le Ménestrel ( 8 mars 1863 p. 109) indiquent la tonalité de sol maj.

Archives

(2) Liste des sources (programmes des concerts Pasdeloup), 1862-1863

Source : https://dezede.org/evenements/id/40442/

Chaque nouvel événement prend place dans un corpus classé chronologiquement dont le nombre d'unités s'élève, au moment de la rédaction de cet article, à plus de quarante mille ${ }^{22}$. Structure du concept Dezède, la liste générale des événements, consultable par le menu "Événements ", est une chronologie athématique dont le principal intérêt est de se livrer à l'exploration libre grâce à l'outil de recherche qui lui est associé (figure 6). 


\section{Recherche libre...}

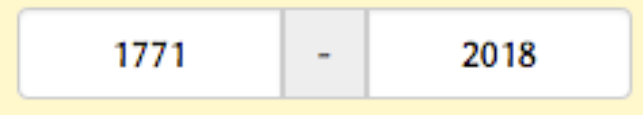

-

\section{Par année civile Par saison}

Lieu...

Euvre...

Individu...

Ensemble...

\section{Filtrer}

Source : https://dezede.org/evenements/

Celui-ci offre autant de possibilités de filtrage des événements visibles qu'il y a de manières de questionner ce corpus, c'est-à-dire, sans limite : outre une rubrique de " recherche libre " qui permet d'interroger toutes sortes de matières (par exemple, une date précise, l'adjectif " romantique ", l'instrument "violon », le prénom " Raymond », mais plus généralement toutes sortes de lieux, de titres, de noms propres), cet outil de recherche avancée dans les événements comporte un instrument dédié au réglage d'une plage de dates par l'intermédiaire d'un double curseur, deux boutons activant un mode " par année civile " ou "par saison ", et quatre rubriques permettant d'atteindre des données exactes hébergées dans la base de données (lieu, œuvre, individu, ensemble) dont la particularité est le caractère cumulatif - par exemple pour sélectionner plusieurs titres d'un même auteur dans trois lieux différents (figure 7). 
FIGURE 7. EXEMPLE DE FILTRAGE DES ÉVÉNEMENTS

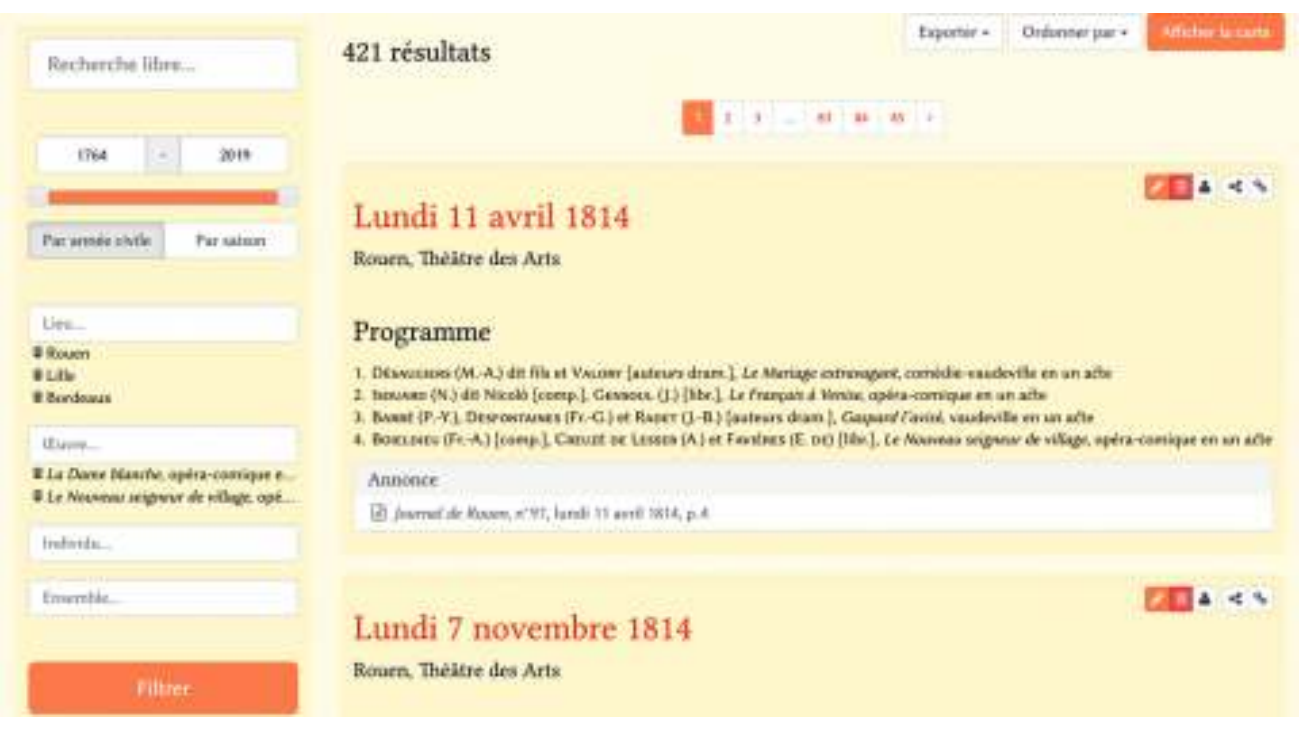

421 résultats (événements) pour deux œuvres de Boieldieu représentées à Bordeaux, à Lille et à Rouen

Source : https://dezede.org/evenements/?

dates_1 $=2018 \&$ lieu $=\% 7 \mathrm{C1} \% 7 \mathrm{C} 84 \% 7 \mathrm{C} 59 \% 7 \mathrm{C} \&$ lieu_text $=\& \mathrm{q}=\&$ dates_0 $=1771 \&$ individu_text $=\&$ oeuvre $=\% 7 C 46 \% 7 C 142 \% 7 C \&$ ensemble_text $=$ \&oeuvre_text $=/$ 


\section{Les dossiers}

La liste générale des événements n'est en rien l'embryon d'un répertoire exhaustif dont les contours évolueraient en permanence en fonction des contributions des utilisateurs. Si chaque nouvel événement créé rejoint cette base générale, il prend un tout autre sens une fois intégré à un ensemble thématique, le "dossier ». Celui-ci est la forme la plus aboutie des possibilités de publication en ligne dans Dezède. Il met à la disposition des chercheurs un modèle de moissonnage automatique des événements à partir d'éléments de paramétrage simples. Ainsi le chercheur " anonyme " ne s'attèle plus à la simple reconstitution d'événements noyés dans la grande chronologie, il signe en tant qu'auteur un dossier sur une thématique précise qu'il produit seul ou qu'il dirige. Ces dossiers sont consultables par le menu « Dossiers » et intègrent une table des matières évolutive en fonction des projets qui comporte aujourd'hui huit entrées : archives du spectacle ; institutions et spectacles ; répertoires de la scène et leur diffusion ; concerts en France ; fêtes, hommages, centenaires et commémorations ; carrières et tournées d'artistes ; musiques actuelles ; partenaires. Il peut s'agir du répertoire d'une institution sur une période précise (l'Opéra-Comique sous la Restauration (Élart 2014b), l'opéra-théâtre de Metz dans l'entre-deuxguerres (Branger 2016) ou le Théâtre des Arts de Rouen entre 1882 et 1940 (Simon 2013), d'une société constituée (les Concerts Lamoureux entre 1881 et 1899 [Simon 2015]), d'un artiste ou d'un ensemble en tournée (le séjour de Cornélie Falcon à Rouen en 1837 [Brunel 2015]) ou les voyages de Monsieur Pasdeloup entre 1864 et 1883 (Simon 2014), de la création d'une œuvre (Samson et Dalila [Élart 2013b], Lohengrin [Simon 2012] ou Le Rêve [Branger 2016]), de sa diffusion dans le temps et dans l'espace (La Traviata en France de 1861 à 1901 [Morvannou 2014], Lohengrin en France en 1891 [Simon 2012], les pièces de Labiche en province entre 1837 et 1888 [Carrère-Saucède 2014], ou les œuvres de Boieldieu en France de la Révolution à la Première Guerre mondiale [Élart 2014c]), ou encore de l'évolution d'une forme d'organisation du spectacle (les concerts dans les résidences royales sous la monarchie de Juillet [Vernet 2014], les fêtes Corneille à Rouen de 1784 à 1884 [Élart 2019], les concerts de rock en France [Élart 2017a]).

Le dossier est introduit par une présentation formatée, imposant un cadre immuable en trois parties : contexte historique, sources et protocole, bibliographie indicative. D'autres éléments éditoriaux s'ajoutent en marge de cette structure principale : l'éditeur scientifique, les éventuels contributeurs (moissonnage automatique par la signature des données), la date de mise en ligne, les publications associées lorsqu'elles existent cette pratique consistant à faire d'un dossier dans Dezède le complément d'une publication papier, ou l'inverse, est vivement encouragée -, les possibles développements envisagés et rebonds vers d'autres dossiers Dezède, et enfin la mention de citation du dossier sous la forme d'une référence bibliographique reconstituée automatiquement ("Pour citer ce dossier »). De plus, un bouton permet d'exporter automatiquement le dossier au format PDF, dont le fichier se télécharge en quelques secondes via l'adresse électronique du compte utilisateur connecté et réfé- 
rencé dans la base de données ; les données du dossier - présentation et événements - sont alors entièrement compilées sous LaTeX pour faciliter en particulier la constitution des annexes d'un travail universitaire.

À la présentation d'un dossier correspond un ensemble d'événements dont le nombre varie entre un et plusieurs milliers, rassemblés dans le troisième et dernier onglet du dossier (voir figure 8, avec ici 143 événements). Entre ces deux onglets, le deuxième donne accès à plusieurs outils de visualisation des données contenues dans le dossier. Ces outils sont actuellement au nombre de quatre et devraient évoluer dans les prochaines années : l'outil cartographique (figure 8) permet de géolocaliser les événements en proposant différentes échelles territoriales (pays, régions, départements, villes, salles) et, en fonction du zoom choisi, des cercles proportionnels aux événements (figure 9) ; l'outil de diffusion (figure 8) propose pour les ensembles une répartition des aires géographiques de diffusion - lieu de résidence, diffusion nationale, diffusion mondiale - commentées, au passage de la souris, par des bulles précisant la nature du territoire, le pourcentage et le nombre d'événements ; l'outil périodique (figure 8) regroupe les œuvres citées dans les événements sous forme de frise chronologique par grande période musicale ; le diagramme des auteurs les plus représentés (figure 10) tire quant à lui des cordes proportionnelles entre les auteurs cités dans les événements, révélant en un coup d'œil les associations récurrentes de répertoire dans les programmes de concert ou de théâtre.

FIGURE 8. DIVERS OUTILS DE VISUALISATION

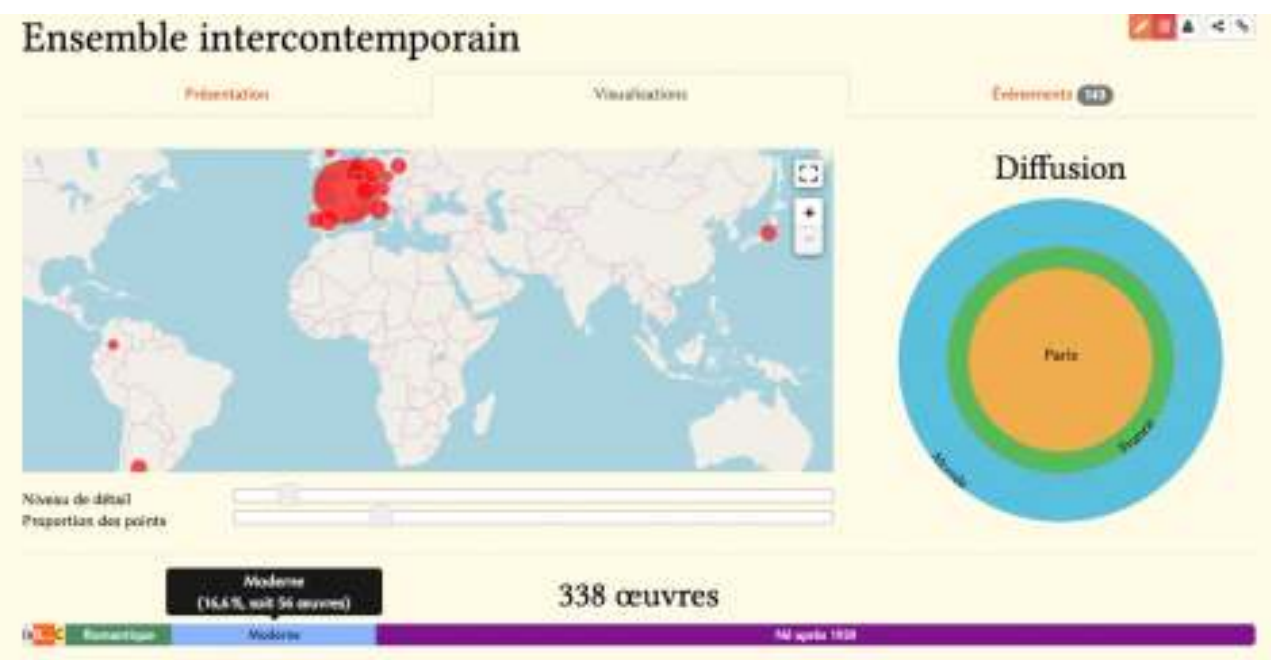

Outils de visualisation appliqués au dossier de l'Ensemble intercontemporain (saisons 2010-2013) : outil cartographique, outil de diffusion, outil périodique

Source : https://dezede.org/dossiers/afo-ensemble-intercontemporain/stats 


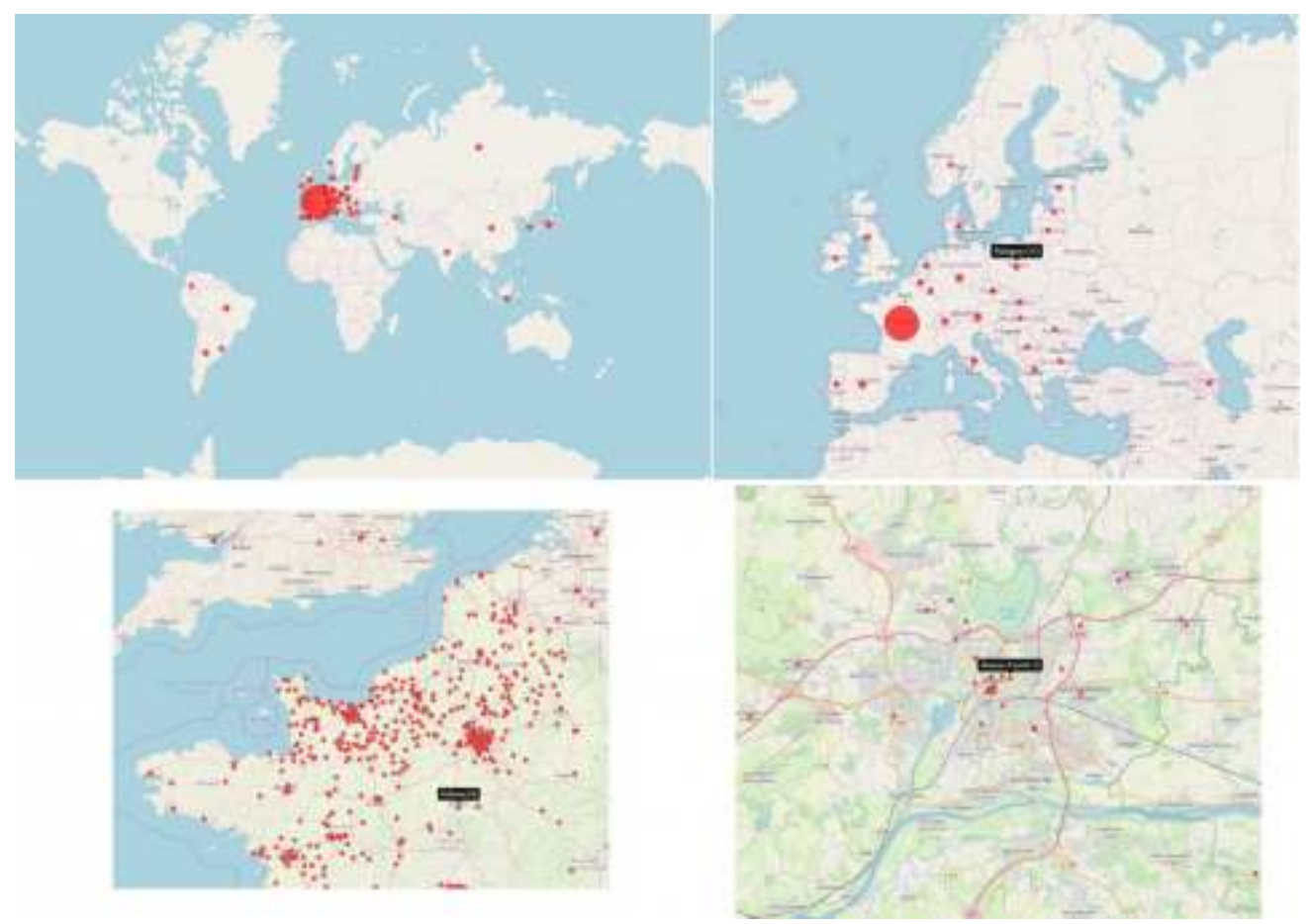

Cartes publiées dans le dossier « Association française des orchestres ", Dezède. L'outil cartographique établit un regroupement des événements par lieu ; doté d'un zoom, il permet de changer d'échelle territoriale (pays, régions, départements, villes, salles) en ajustant les cercles proportionnels en fonction du nombre d'événements.

Source : https://dezede.org/dossiers/afo/stats 


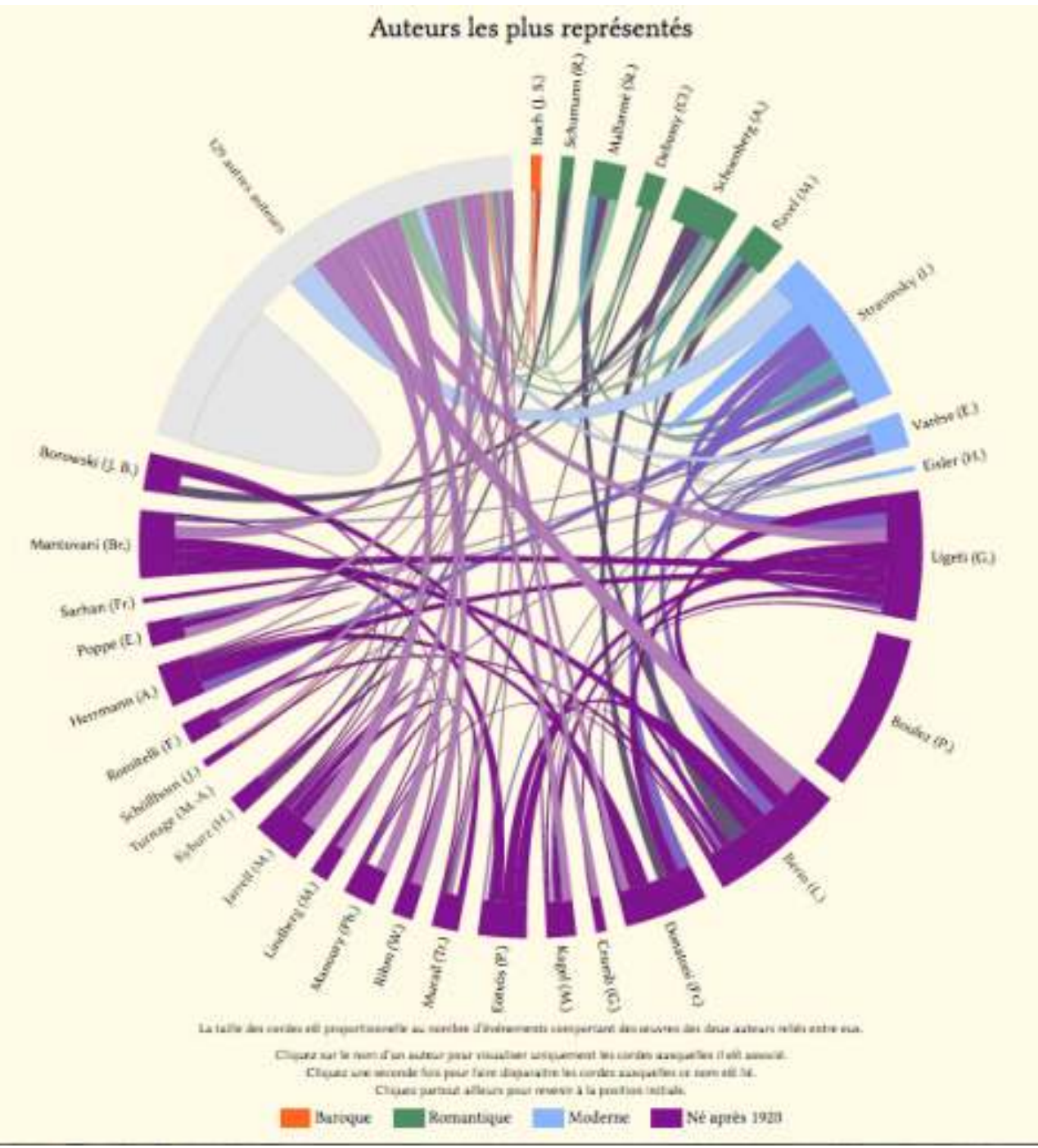

Outils de visualisation appliqués au dossier de l'Ensemble intercontemporain (saisons 2010-2013) : diagramme de cordes des auteurs les plus représentés dans le dossier Source : https://dezede.org/dossiers/afo-ensemble-intercontemporain/stats 


\section{Les sources}

Adossée aux pratiques des historiens, la méthode Dezède consiste à reconstituer des événements à partir de sources variées. Contrairement à leurs transcriptions «brutes » que nous avons évoquées en première partie, les événements dans Dezède sont des reconstructions appuyées sur la confrontation des sources et l'accumulation des connaissances des porteurs de projet. L'objectif de l'enquête historique est de parvenir à une identification la plus précise possible du programme de l'événement, pour en constituer, en quelque sorte, une autorité. Il ne s'agit pas simplement de peindre à grands traits l'histoire des spectacles, mais d'entrer dans le détail de chaque programme autant que possible : l'ordre d'interprétation des œuvres, la précision du fragment d'une œuvre quand elle n'est pas jouée en entier (une ouverture, un air, un mouvement, etc.), de la version quand il en existe plusieurs (arrangement, orchestration, transcription, etc.) ou de la version utilisée pour les œuvres vocales (version originale, traductions), la distribution des interprètes, et l'apparat critique signalant des éléments particuliers que le modèle de présentation ne permet pas d'introduire (figure 5). Les types de sources et leurs modes d'accès sont multiples sur Dezède. A minima, il s'agit d'une cote indiquant le lieu de conservation de la source ou identifiant la livraison d'un périodique. Les articles de presse peuvent faire l'objet d'une transcription intégrale dont la réunion constitue un dossier de presse favorisant la recherche en plein texte. Des références peuvent être associées à un lien hypertexte pérenne donnant accès à une page Web et à une ressource numérique. Le modèle de gestion des sources donne également la possibilité de télécharger directement une reproduction de la source dans les différents formats usuels (JPG, PNG, etc.), pour l'associer, in fine, à un feuilleteur (voir le dossier des archives de l'Opéra-Comique qui inaugure le premier dossier de sources dont le principe est rendu générique en 2019 avec la "bibliothèque " [Opéra-Comique 2014] ou des concerts de Royaumont [Élart et Simon 2013c]). Par ailleurs, pour les corpus de spectacles récents, les sources peuvent être des fichiers audio ou vidéo téléchargés. Le choix des sources est conditionné par leur accessibilité mais aussi par les objectifs poursuivis par les éditeurs scientifiques ou les partenaires.

\section{Le moteur de recherche}

Pour interroger à tout moment le corpus hébergé dans Dezède - les événements, les dossiers, les sources, mais également toutes les autorités disponibles (voir la section suivante) -, il suffit d'utiliser le moteur de recherche mis à disposition dans la barre de menus en en-tête. L'interrogation se fait en deux temps : elle commence par une recherche libre et simple, qui consiste à taper un ou plusieurs mots significatifs de la requête dans la rubrique principale (figure 11). 


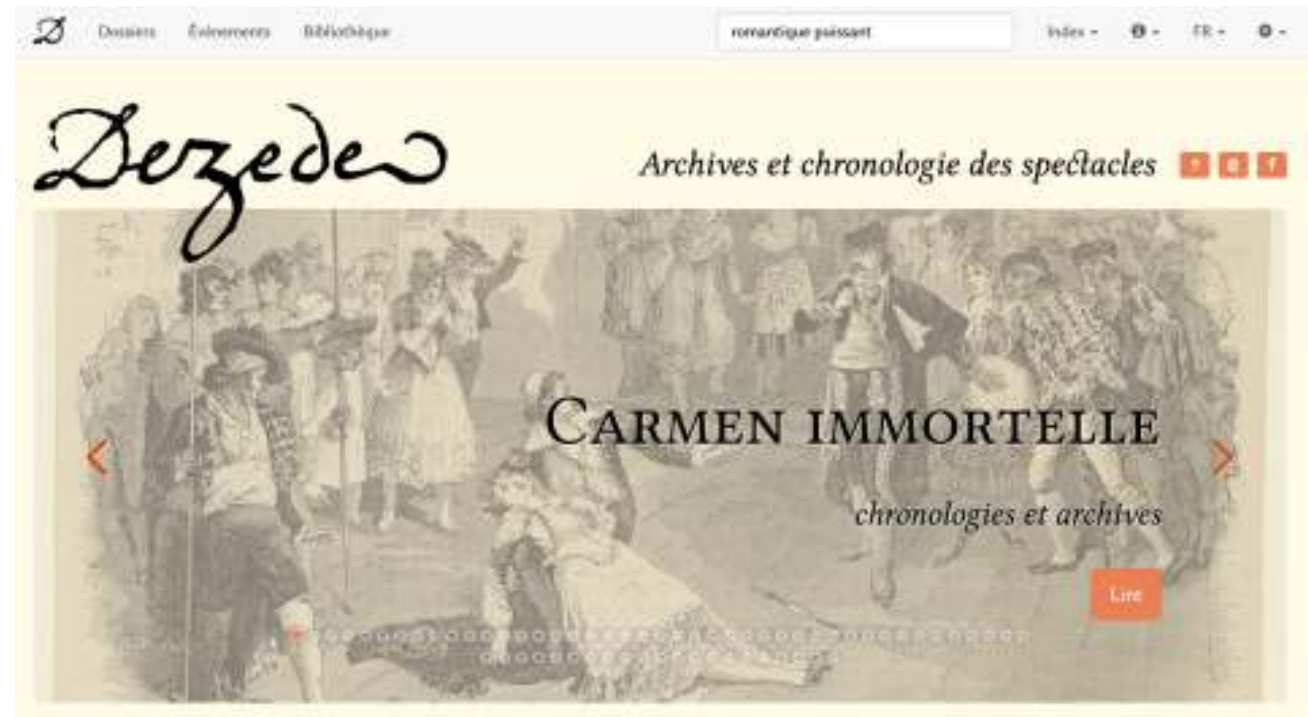

Recherche simple dans Dezède ou première phase de lancement de requête dans le moteur de recherche (dans notre exemple, " romantique puissant »)

Source : https://dezede.org

Puis, une fois validée, elle conduit l'internaute vers la liste des résultats (figure 12), qui s'affichent de manière synthétique dans le module de recherche avancée. Ce dernier propose un ensemble de filtres par type de données qu'il est possible de combiner entre eux pour affiner la recherche (dossiers d'événements, ensembles, individus, lieux, et institutions, professions, rôles et instruments, sources, événements, œuvres).

FIGURE 12. RECHERCHE AVANCÉE

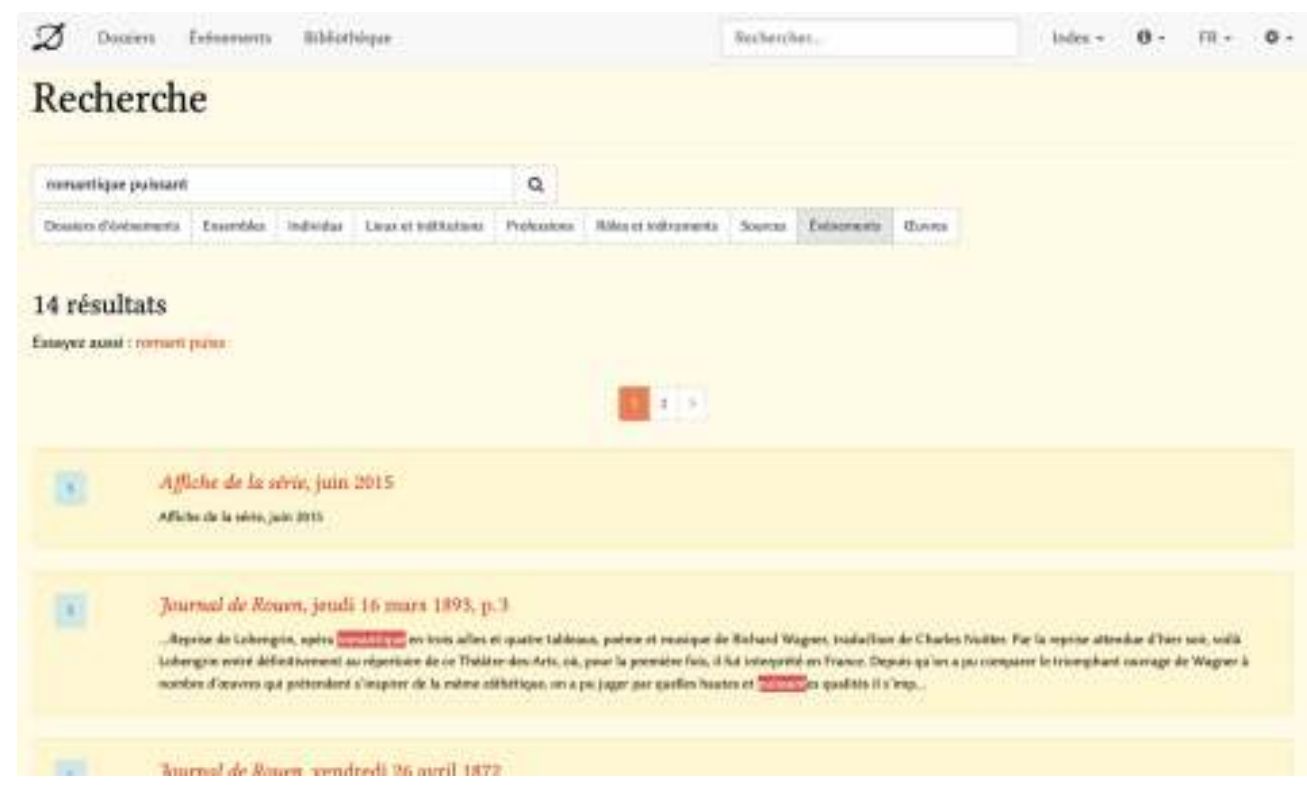

Recherche avancée dans Dezède ou seconde phase pour affiner une recherche (dans notre exemple, "romantique puissant »)

Source : https://dezede.org/recherche/?q=romantique+puissant

En marge de chaque résultat, une icône carrée avec initiale précise le type de données trouvées (ici, un " $\mathrm{S}$ » comme " source »). Il suffit enfin de cliquer sur le lien supérieur du résultat pour consulter la donnée recherchée. 
Le moteur de recherche peut également suggérer des résultats exacts qui répondent à une requête libre : dans notre exemple (figure 13), la recherche au mot " dame " fait apparaître l'autorité de La Dame blanche, qui peut être atteinte, comme toutes les autorités, sans passer par le module de recherche avancée. Cet outil suggère de lui-même des résultats approximatifs comme la ville de Damas ou le compositeur Émile Damais.

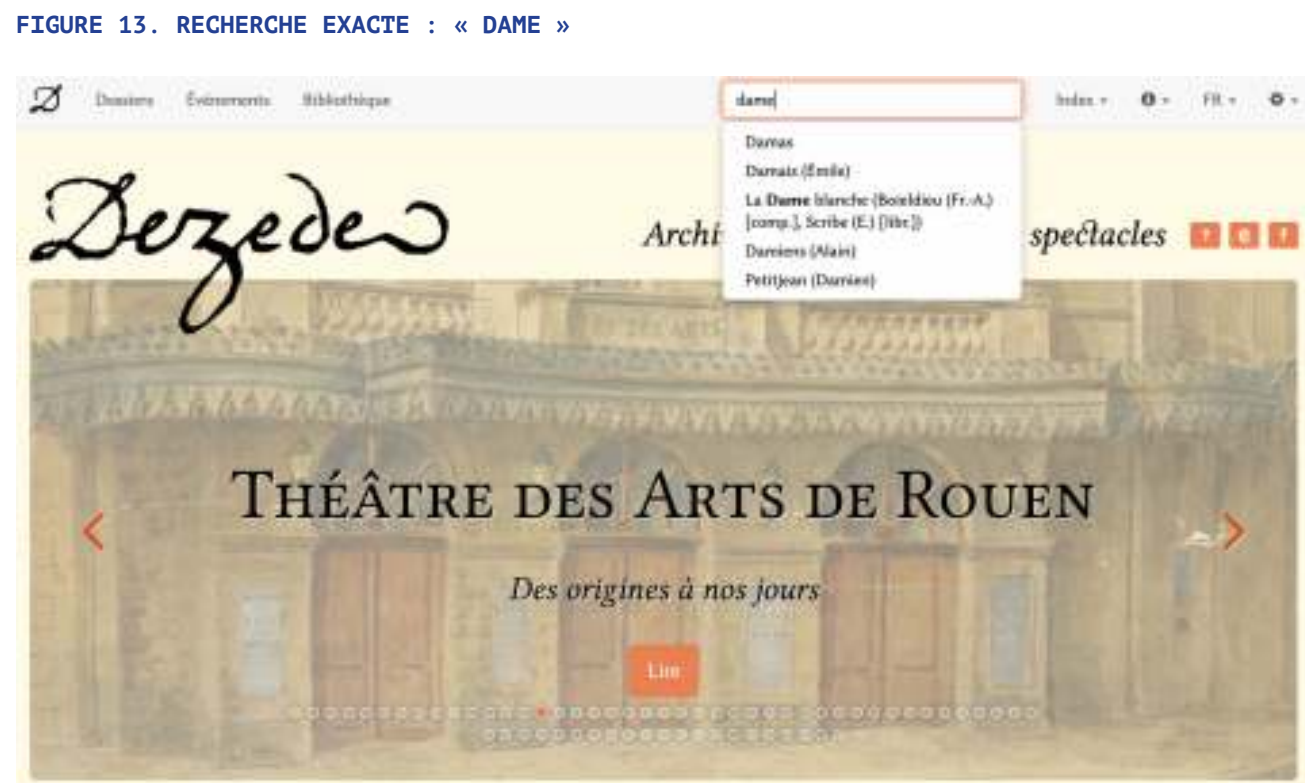

Recherche exacte à «dame » dans Dezède pour retrouver l'autorité de La Dame blanche Source : https://dezede.org

Remarquons enfin que le moteur de recherche réagit différemment à la formulation des requêtes : taper la requête "la dame " génère des réponses privilégiant en majorité, par association de l'article défini, les titres d'œuvres (figure 14) : La Dame du lac en réponse exacte, mais également Les Dames à la mode et La Damnation de Faust en réponses approximatives.

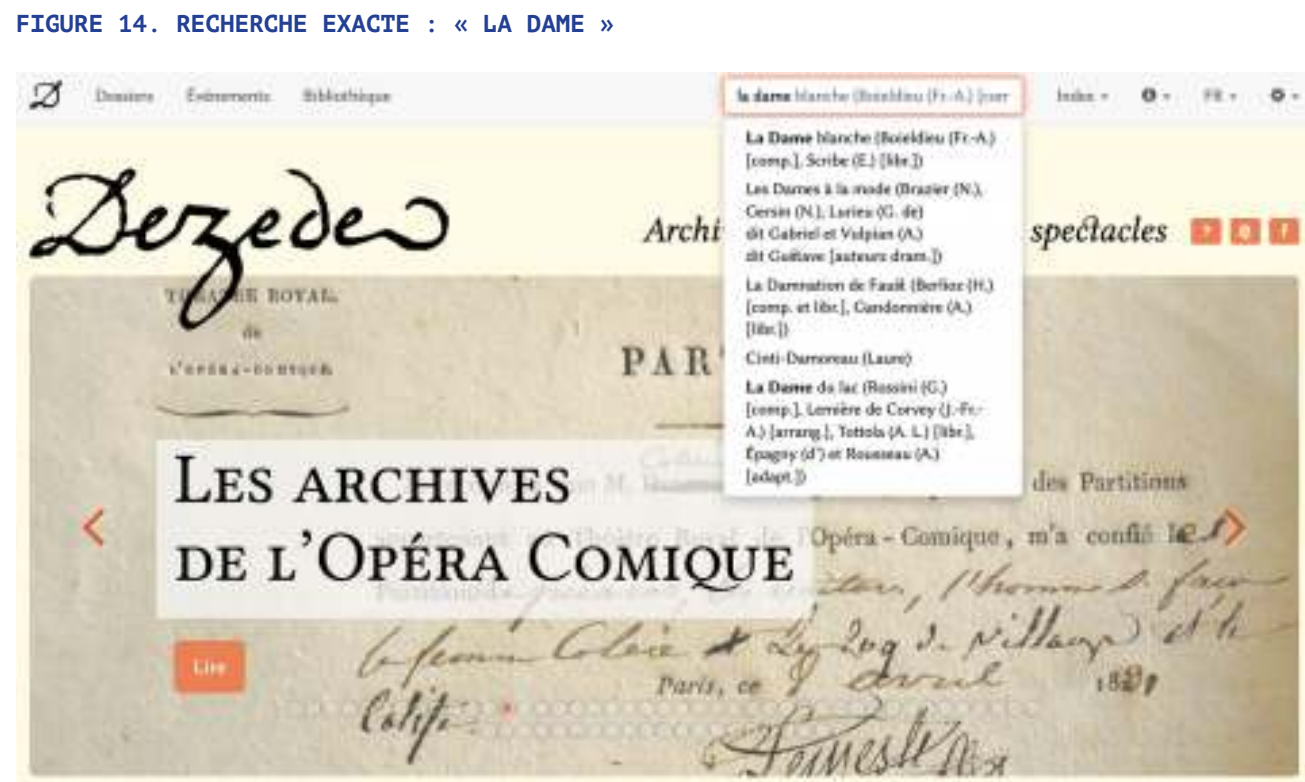

Recherche exacte à «la dame » dans Dezède pour retrouver l'autorité de La Dame blanche Source : https://dezede.org 


\section{Au cour du système : les autorités}

Le cœur de Dezède est doté d'une base de données relationnelle, qui lie chaque événement à un ensemble d' " autorités " disponibles sur les œuvres, les individus, les ensembles, les lieux de diffusion, les rôles, les instruments, les professions, etc. La base gère par ailleurs les relations entre différentes données produites en marge des autorités, à savoir entre les 45890 événements - dont 34530 rendus publics ${ }^{23}$-, entre une partie de ces événements et les 296 dossiers - dont 222 rendus publics -, ou encore entre les événements et les 57928 sources. Du point de vue des autorités à proprement parler, Dezède dispose, actuellement, d'un référencement pour 22320 œuvres, 15838 individus et 7719 lieux. Ces trois ensembles de données constituent la matière de base pour la fabrication des événements, matière qui est également partagée entre tous les utilisateurs quel que soit le type de compte. L'architecture de Dezède est par ailleurs conçue pour que ces autorités puissent dialoguer entre elles : à partir du mode de consultation principal, les événements, il est en effet possible de « rebondir " sur les données liées, en consultant la fiche de tel artiste, de telle œuvre ou de tel lieu. Toutes les unités d'information présentées dans l'événement sont ainsi dotées d'hyperliens qui favorisent le rebond et la consultation sans fin : ainsi, à partir d'une fiche d'individu, il est possible de consulter tous les événements liés, une partie des événements liés, une œuvre en particulier ou un individu parent. Toute la complexité de la gestion des chronologies événementielles est ici résolue dans le programme de Dezède, qui est parvenu à marier des données diverses sans aucune redondance, avec une grande souplesse et une infaillible rigueur d'écriture. Il y est parvenu en s'appropriant le principe des identifiants pérennes dont l'incrémentation, propre à ce système, est automatique - à chaque donnée correspond un numéro d'identifiant unique. Enfin, le corpus d'autorités enrichi chaque jour par les contributeurs puise ses origines dans des travaux antérieurs qui ont fait l'objet d'importations automatiques ${ }^{24}$. Elles sont produites ou vérifiées à partir d'un ou plusieurs outils bibliographiques mentionnés dans la bibliographie générale du site ${ }^{25}$. Toutes les références à des sources ou à des ouvrages non mentionnés sont signalées directement dans les notes privées des données concernées.

Du point de vue de la constitution, la nature de ces autorités diverge quelque peu des normes usuelles de la Bibliothèque nationale de France, car elle est adossée à la réalité des spectacles, et non à celle des éditions des œuvres interprétées, même s'il faut reconnaître qu'elle doit beaucoup aux méthodes adoptées par les bibliothécaires. Chaque donnée est référencée par une fiche qui lui est propre, selon un modèle immuable en fonction du type de données, chaque autorité contient en général une liste des événements dans lesquels elle apparaît. Il est ainsi possible d'accéder à tous les événements contenant le titre d'une œuvre, le nom d'un compositeur ou d'un interprète, celui d'une ville ou d'une salle. Chaque autorité peut être intégrée dans un ensemble de données récursives (dynasties de musiciens, adaptations et révisions d'œuvres, rattachements des lieux aux territoires et des salles aux institutions, etc.) Arrêtons-nous sur deux modèles en particulier : les individus et les œuvres. 
Pour chaque individu (figure 15), le prénom en bas de casse et le patronyme en petites majuscules - dans cet ordre et non dans l'ordre inverse, comme dans la plupart des bases de données prosopographiques -, sont suivis des lieux et dates de naissance et de décès - au jour près -, des professions, puis de listes moissonnées automatiquement d'œuvres et d'événements présents dans Dezède, introduites par des sommes calculées automatiquement ; enfin, le modèle permet de lier des parents entre eux, comme c'est le cas pour Mozart et son père dans notre exemple ${ }^{26}$, de gérer les noms de naissance et certains pseudonymes.

FIGURE 15. NOTICE D'AUTORITÉ D'UN COMPOSITEUR

\title{
Wolfgang Amadeus MozART
}

\author{
Salzbourg, 27 janvier 1756 - Vienne, 5 décembre 1791 \\ Compositeur et arrangeur
}

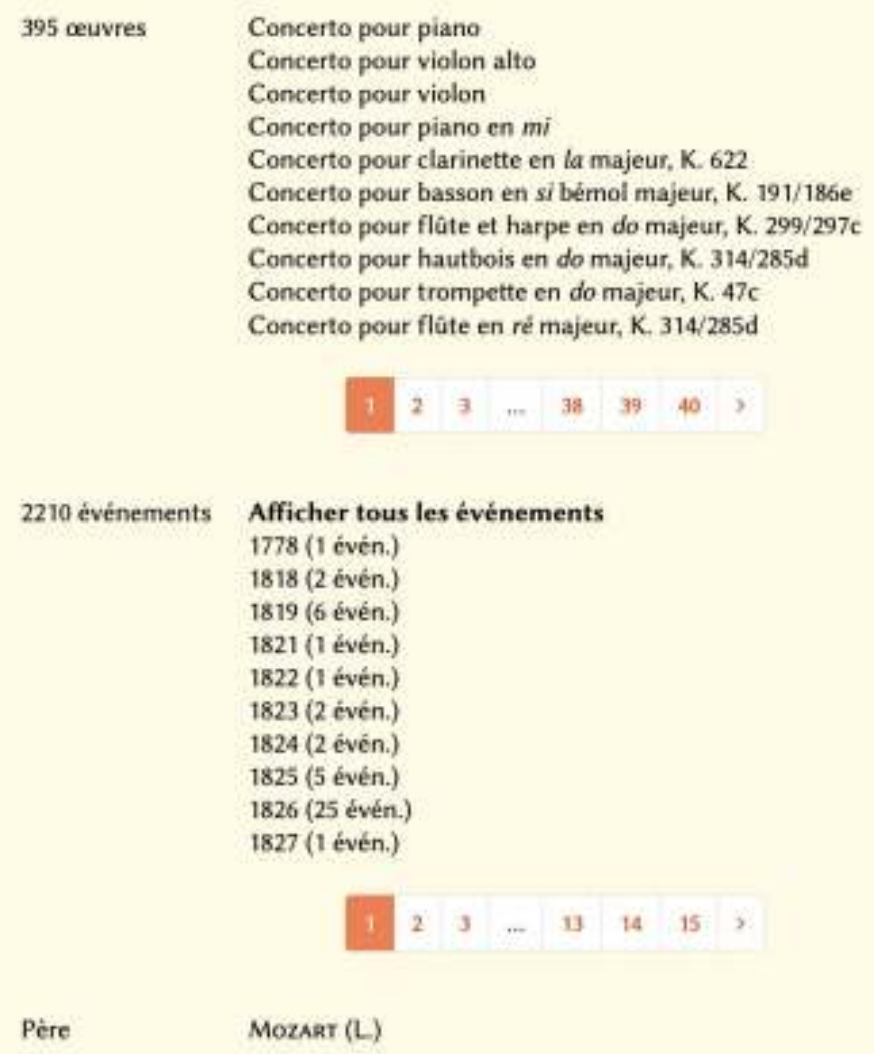

Autorité « Wolfgang Amadeus Mozart » dans Dezède

Source : https://dezede.org/individus/id/330/

Pour les œuvres, le modèle de gestion permet de produire deux formes d'autorités, qui respectent les normes sur les titres significatifs (Don Giovanni) et non significatifs construits sur les genres (Symphonie $n^{0} 31$ ). Toutefois, ces deux formes suivent la même présentation (figure 16) : au titre en italique - auquel peut être adjoint un ensemble de qualificatifs (titre secondaire, surnom, opus, indicatif au catalogue thématique, numéro d'œuvre, tonalité, incipit, arrangement, etc.) -, s'ajoutent le genre et la coupe, puis le ou les auteurs sous une forme synthétisant et remodelant automatiquement les autorités individus suivies de leur profession dans l'œuvre en question, le lieu et la date de création. Il est 
également possible de reconstituer la distribution d'exécution de l'œuvre (rôles et pupitres). Le modèle permet par ailleurs de retrouver tous les liens de parenté entre les œuvres, quand elles dérivent d'une version antérieure (arrangement ou simple inspiration) ou quand elles sont traduites. Sont publiées enfin des listes moissonnées automatiquement, présentant les différents événements de l'œuvre reconstitués dans $D e$ zède, les sources numérisées hébergées dans Dezède et un outil ingénieux de calcul automatique du nombre d'associations de l'œuvre avec d'autres œuvres du corpus d'événements, présentées dans un ordre numérique inversé.

FIGURE 16. NOTICE D'AUTORITÉ D'UNE EUVRE

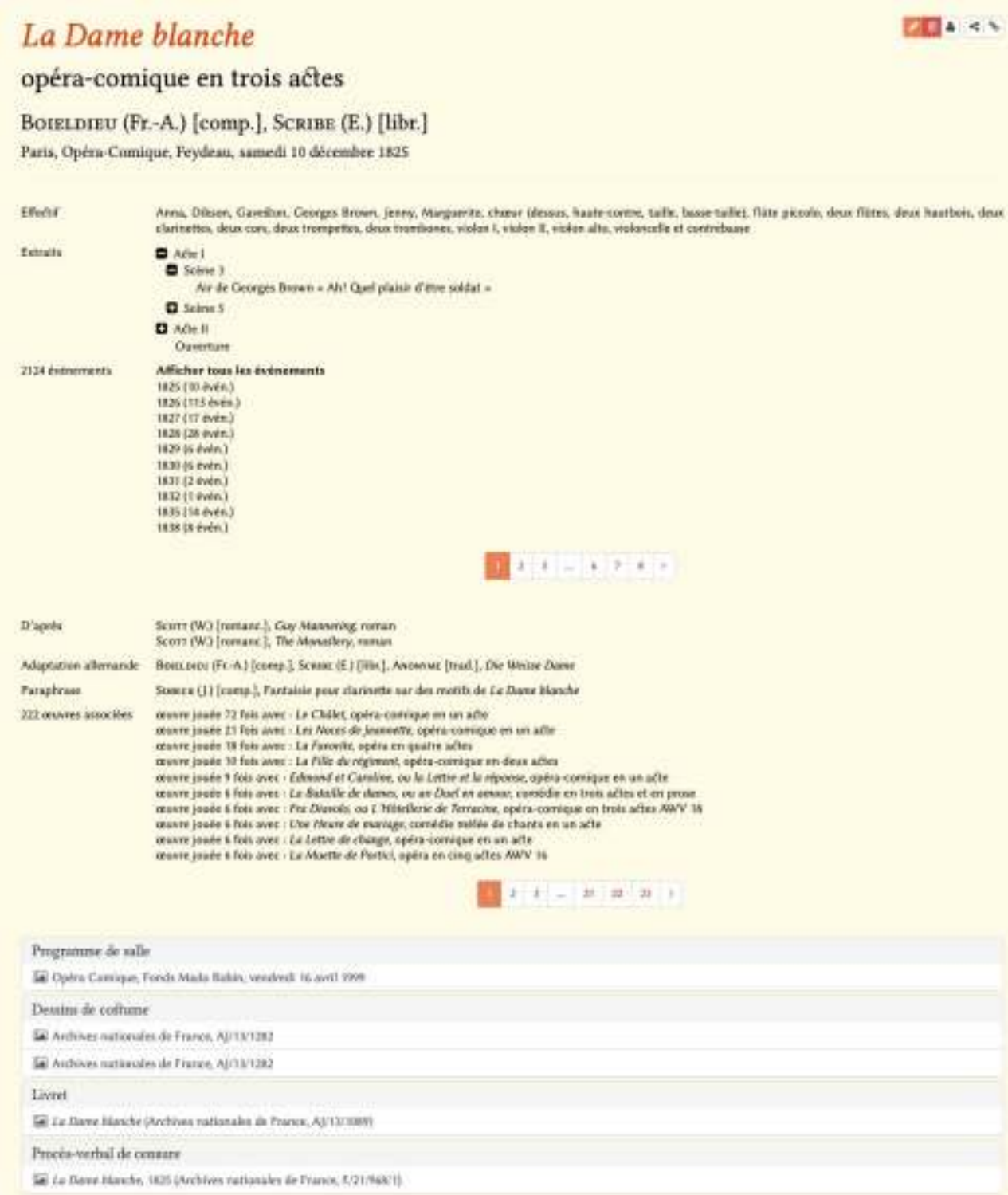

Autorité de La Dame blanche publiée dans Dezède

Source : https://dezede.org/oeuvres/id/46/

Le dernier élément constitutif du modèle de gestion des œuvres, probablement le plus avancé dans le domaine de la chronologie événementielle, est celui des extraits : dans notre exemple (figure 17), l'extrait peut être une ouverture, un acte, une scène ou un air. Comme la vocation de Dezède est la reconstitution de spectacles, et comme plusieurs formes de spectacle ont recours à des extraits d'œuvres, Dezède devait être en capacité de gérer ces fragments. De ce point de vue, Dezède se distingue des 
catalogues de bibliothèques qui ignorent les extraits d'œuvres sauf s'ils ont fait l'objet d'une édition séparée. L'exemple ci-dessous est très représentatif du mode de gestion des extraits dans Dezède et de ses possibilités multiples.

FIGURE 17. NOTICE D'AUTORITÉ D'UN AIR

\section{Les Maîtres chanteurs de Nuremberg, III, 5, Air de Walther (FR Ernst) « L'Aube vermeille brillait dans les cieux »}

Chant de concours

WAGNER (R.) [comp, et libr.], ERNST (A.) [adapt.]

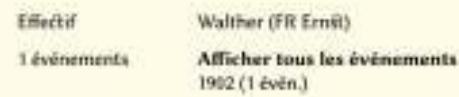

Autorité de l'air $\mathrm{n}^{0}{ }_{5}$, «L'Aube vermeille brillait dans les cieux », extrait du troisième acte des Maîtres chanteurs de Nuremberg dans la version française d'Alfred Ernst

Source : https://dezede.org/oeuvres/id/28357/

L'autorité s'applique à l'air « L'Aube vermeille brillait dans les cieux ». À partir de cette autorité, il est possible de rebondir vers la scène contenant l'air (" 5 »), l'acte ("III ») et l'œuvre ("Les Maîtres chanteurs de Nuremberg »). En l'occurrence, celle-ci n'est pas le dernier maillon de la chaîne avant l'autorité ultime du compositeur, Les Maîtres chanteurs de Nuremberg étant l'une des adaptations françaises de l'œuvre originale composée par Richard Wagner, Die Meistersinger von Nürnberg. L’ouvre mère est elle aussi accessible depuis l'autorité de la version française (figure 18).

FIGURE 18. NOTICE D'AUTORITÉ D'UNE VERSION D'UNE EEUVRE

\section{Les Maîtres chanteurs de Nuremberg opéra en trois actes}

WAGNER (R.) [comp. et libr.], ERNST (A.) [adapt.]

\begin{tabular}{|c|c|}
\hline Extraits & $\begin{array}{l}\text { ( Acte I } \\
\text { ( Acte III }\end{array}$ \\
\hline 9 évènements & $\begin{array}{l}\text { Afficher tous les événements } \\
1895 \text { ( } 4 \text { évén.) } \\
1902 \text { ( } 1 \text { évén.) } \\
1910 \text { ( } 1 \text { évén.) } \\
1911 \text { ( } 1 \text { évén.) } \\
1913 \text { ( } 1 \text { évén.) } \\
1914 \text { ( } 1 \text { évén.) }\end{array}$ \\
\hline $\begin{array}{l}\text { Version française de } \\
6 \text { ceuvres associées }\end{array}$ & 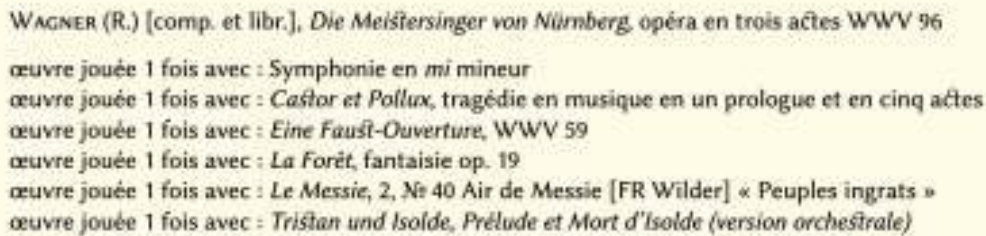 \\
\hline
\end{tabular}


Le modèle de gestion des œuvres permet ainsi d'appliquer le principe des autorités de la même manière aux œuvres, à leurs versions et à leurs fragments. Elle met en relation des unités d'information - un mouvement de symphonie, un air, un acte, un opéra, etc. - ayant été considérées, au moins une fois, comme un élément de programme à l'intérieur d'un événement.

Pour terminer, précisons que Dezède, sans être encore interopérable ${ }^{27}$, intègre deux référentiels internationaux. Le premier est l'ISNI - International Standard Name Identifier -, qui est un code international normalisé servant à identifier les "identités publiques des personnes ou des organismes impliqués dans la création, la production, la gestion et la distribution de contenus intellectuels et artistiques ou faisant l'objet de ces contenus " (ISNI s.d.). Cette identification " univoque, sur le long terme et à l'échelle internationale " (ISNI s.d.) facilite un dialogue sans faille entre bases de données exploitant ce système géré par une agence internationale (ISNI-IA), dont sont membres la Bibliothèque nationale de France ou la British Library. Le second référentiel est OpenStreetMap : intégré au modèle de gestion des lieux, il permet de géolocaliser les pays, les villes et les autres niveaux d'administration territoriale, mais également les salles et les institutions. Ce système a une répercussion directe, puisqu'il est à la base de la gestion des représentations cartographiques dans Dezède.

Les autorités de Dezède composent donc le cœur stratégique de la base de données. Elles font l'objet d'un contrôle permanent et de mises à jour régulières. Les ajouts et les corrections sont consignés dans un historique des versions, et peuvent être commentés dans une rubrique de « notes privées " dans lesquelles des références bibliographiques précisent les sources d'information.

\section{La communauté Dezède}

\section{Des chercheurs aux étudiants}

Dezède est mis à la disposition des chercheurs et des étudiants qui souhaitent publier des corpus documentaires sur un artiste, une œuvre, un lieu de spectacle, un phénomène musical ou théâtral. Modélisé dans un esprit de pluridisciplinarité, Dezède s'adresse aux chercheurs inscrits dans différents champs disciplinaires : musicologie, lettres modernes, histoire culturelle moderne et contemporaine, arts du spectacle, histoire de l'art, histoire de la danse, etc. Il propose également aux jeunes chercheurs inscrits en master ou en doctorat une aide à l'édition - mise à disposition des autorités, module d'exportation des annexes avec mise en page automatique - et à la valorisation des travaux universitaires confidentialité des données personnelles, possibilité de différer la publication en ligne - qui, une fois publiés, valent comme références bibliographiques. Tourné vers le développement de pratiques pédagogiques innovantes, Dezède est également un support pour l'enseignement universitaire, en particulier de la méthodologie et des technologies de l'information (voir la section suivante).

Chaque utilisateur, qu'il soit étudiant, chercheur ou partenaire, est propriétaire de ses données. En les produisant à partir de son compte, 
l'utilisateur en devient automatiquement l'auteur et le propriétaire. Cet affichage offre une transparence totale sur l'appartenance et la provenance des données, même si la forme de ces dernières n'est pas figée et est susceptible d'être modifiée par l'équipe éditoriale. La traçabilité des données passe par la constitution d'un compte utilisateur, qui est validé une fois le projet du porteur accepté par le comité éditorial. Le nouvel utilisateur est alors accompagné par un utilisateur confirmé dans une première phase de formation, qui peut être organisée en groupe à l'université de Rouen (deux formations en moyenne par an). La traçabilité des données facilite par ailleurs toutes les opérations pour leur maintenance.

\section{Des partenaires}

Au nombre des membres de la communauté Dezède, il faut compter les partenaires. Dezède est une solution d'archivage et de promotion du spectacle vivant susceptible d'intéresser les institutions souhaitant valoriser leur patrimoine, par exemple sous la forme d'une banque d'images - programmes de salle, documents iconographiques, etc. - ou d'un répertoire d'événements élaboré suivant les critères scientifiques définis plus haut. À partir de 2013, quatre partenariats ont été initiés et ont donné lieu à la signature d'une convention. Le premier a consisté à mettre en ligne sur Dezède les archives de l'Opéra-Comique (Opéra-Comique 2014) dans le cadre du tricentenaire de l'institution. Issus de ses propres archives ou de celles conservées par les Archives nationales de France, un peu plus de deux mille documents iconographiques - soit près de 22 ooo images numériques présentées en feuilleteur - sont désormais accessibles suivant deux modes de lecture (par ordre alphabétique des ouvrages ou chronologique de leur première représentation). Ce sont des livrets manuscrits, des dossiers de censure, des affiches, des programmes de salle, des brochures de saison, des dessins, des gravures, des photographies couvrant la période de 1800 à 2007. Contrairement au précédent dont la réalisation est achevée, le deuxième est en cours de réalisation. Il permet à des étudiants du master de musicologie de l'université de Rouen de participer à la réalisation du dossier réunissant les programmes des concerts organisés à la Fondation Royaumont (Élart et Simon 2013C) à partir de 1936. Chaque année depuis 2013, un groupe d'étudiants est reçu à l'abbaye de Royaumont pour un séjour de travail destiné à contribuer à ce projet dont l'aboutissement n'est pas encore en vue. Le troisième est un vaste chantier consistant à rassembler sur Dezède les programmes des saisons de concerts et les archives audiovisuelles des orchestres membres de l'Association française des orchestres ( Association française des orchestres » 2014). Ce projet très ambitieux d'archiver presque en temps réel l'activité d'une quarantaine de formations orchestrales professionnelles est malheureusement à l'arrêt présentement, même si un projet récent (CORNUM, 2019-2022) tend à réactiver le volet des orchestres normands. En effet, un quatrième partenariat signé entre l'université de Rouen Normandie et l'opéra de Rouen Normandie vise précisément à reconstituer l'activité complète de son orchestre depuis sa fondation en 1998 jusqu'à nos jours. 


\section{L'enseignement}

Depuis une vingtaine d'années, l'enseignement de la méthodologie en licence et en master de musicologie à l'université de Rouen est adossé à un projet de recherche, la chronologie événementielle, dont le principe est fondé sur la reconstitution des spectacles à partir de sources. L'objectif est de familiariser les étudiants avec les sources, leur maniement et leur transcription, ou avec la constitution d'autorités. Toutes ces expériences sont réalisées dans le cadre d'un projet de recherche d'une dimension adaptée à la durée du cours ou d'une ampleur beaucoup plus large mais au sein duquel les étudiants sont amenés à apporter leur contribution. Avant la création de Dezède, ce travail de sensibilisation à la recherche était réalisé à la main, dans un premier temps sur des fiches cartonnées, puis, dans un second temps, avec l'aide d'un outil de traitement de texte. Mais l'inauguration de Dezède en 2012 a bouleversé cette approche pédagogique. En effet, cet outil de recherche a également été pensé dès la rédaction des premières lignes de code comme un outil de formation. Nourrie par les expériences du passé, cette réflexion a pu profiter d'expérimentations nouvelles menées en 2012 et 2013 aux universités de Rouen et Paul-Valéry-Montpellier 3. C'est ainsi que quatre groupes d'étudiants, de la licence 3 au master 2, ont été autorisés à utiliser le prototype de Dezède dans le cadre de cours de méthodologie universitaire et de préparation au Certificat informatique et Internet (C2i). Il s'agissait pour eux de se former à la méthode de transcription et d'analyse des sources, en utilisant les outils déjà mis à leur disposition sur Dezède, à partir de comptes utilisateurs personnalisés en lien avec celui de l'enseignant. Les quatre objectifs atteints étaient les suivants : l'autonomie dans la recherche documentaire ; la formation aux technologies de l'informatique et l'accompagnement de l'enseignement de $\mathrm{C} 2 \mathrm{i}$; le traitement et l'analyse d'un petit corpus de sources à transcrire ; la gestion d'un projet de recherche en équipe sur la diffusion du répertoire théâtral en province et l'apprentissage de la recherche collective. Pour conclure, cette première expérience de cours "dématérialisé " imaginée sur des objectifs de recherche concrets a permis d'améliorer la formation des étudiants, de les sensibiliser et de les initier à la recherche, de centraliser et d'héberger les résultats, d'accompagner l'édition et la valorisation de leurs travaux.

Une seconde phase d'innovations pédagogiques a été lancée entre 2014 et 2016, grâce à une dotation de l'université de Rouen. Elle a conduit tout d'abord à améliorer et à simplifier significativement plusieurs modèles centraux du back office (sources, événements, individus, œuvres). Cette simplification a consisté en une fusion de sous-modèles vers un modèle unique, autrement dit, en un regroupement d'actions éparpillées sous forme de fenêtres surgissantes ( $p o p-u p$ ) en une seule étape. Durant ces deux années, nous avons pu mesurer le bénéfice de cette amélioration en constatant que les étudiants étaient plus autonomes face au logiciel, qui semblait à l'évidence plus intuitif, plus ergonomique, plus interactif, qu'ils commettaient beaucoup moins d'erreurs de manipulation et qu'ils étaient deux fois plus productifs que les deux années précédentes. Cette dotation a encouragé également le développement d'un module d'exportation des dossiers d'événements au format PDF avec mise en page géné- 
rée automatiquement par LaTeX, outil que nous avons évoqué plus haut. L'étudiant devait pouvoir récupérer les données saisies en ligne pour différents usages (archivage personnel de son travail, impression, insertion en annexe d'une synthèse ou d'un mémoire de master). Enfin et surtout, la dotation a motivé l'invention d'un outil d'autoformation à la transcription diplomatique étape par étape, accessible sans compte utilisateur à l'adresse https://dezede.org/examens/source. Celui-ci propose, dans le scénario actuellement en ligne, de se former en dix étapes progressives à la transcription de sources périodiques (respect de l'orthographe, des ponctuations, des graphies fautives, des styles, etc.) ${ }^{28}$.

Plus précisément, il s'agit de transcrire un texte à partir d'images projetées. Chaque étape est validée lorsque le texte saisi correspond exactement au texte contenu dans l'image de la source. Le tutoriel réagit alors en fonction des problèmes de transcription, en demandant à l'utilisateur de l'améliorer, ou, en cas de réussite, en le guidant à l'étape suivante. Pour connaître la nature des problèmes rencontrés, il suffit de passer la souris sur les encadrés rouges pour faire apparaître une bulle d'aide qui indiquera alors quoi faire. Voyez l'exemple ci-dessous (figure 19) : la bulle d'aide signale un «caractère erroné " pour le mot «Thomas " qui a été saisi par erreur à la place du mot «Pierre ».

FIGURE 19. OUTIL D'AUTOFORMATION À LA TRANSCRIPTION

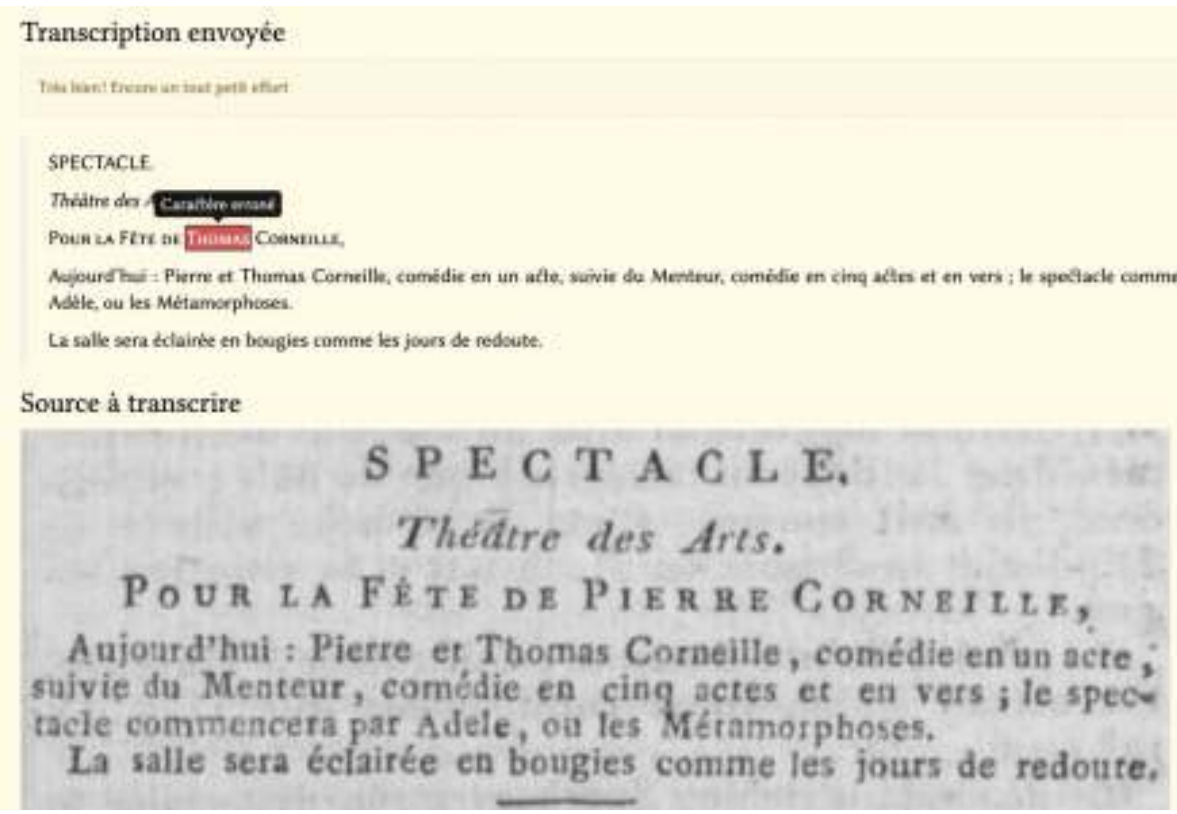

Outil d'autoformation à la transcription diplomatique dans Dezède

Source : https://dezede.org/examens/source

Cette approche pédagogique innovante est basée sur les critères de la recherche collective, collaborative et contributive, qui responsabilise l'étudiant à deux niveaux : à titre personnel, puisqu'il est responsable de son corpus ; au titre du groupe, puisqu'il apporte une contribution à un ensemble qui devient cohérent. Associés ainsi à un projet collaboratif, les étudiants sont donc plus souvent contributeurs que simples récepteurs, le caractère collaboratif étant lié à la mise en commun de résultats à l'intérieur d'une promotion et entre promotions d'un niveau d'étude à l'autre, voire d'une année à l'autre. Sous ces aspects, Dezède trouve parfaitement sa place dans l'ensemble des nouvelles pratiques d'enseignement visant à faire de l'étudiant l'acteur de sa propre formation, entre 
pédagogie inversée et pédagogie participative. Du reste, si l'étudiant est connecté au groupe, il existe bel et bien en tant qu'individu dans le groupe : en signant chaque donnée qu'il produit - la signature est attribuée automatiquement par les identifiants du compte utilisateur -, son nom est ajouté dans la liste des contributeurs du projet accueilli au sein d'un dossier. À titre d'exemples, certains dossiers comme " Le Ring à Rouen (1927) " ont été réalisés au cours d'un semestre, mais d'autres comme "Le Théâtre des Arts de Rouen des origines à nos jours " ou "L'Opéra-Comique à travers les siècles » sont périodiquement inscrits au programme. Une troisième catégorie de dossiers est de nature plus expérimentale et porte sur des territoires encore inédits, comme les dossiers "Maîtrise de Radio France " sur le répertoire choral, "Monsigny en France " sur le traitement d'une source comptable - les registres de la Société des auteurs et compositeurs dramatiques -, "Spectacles à Paris " sur une approche transversale de l'offre théâtrale parisienne à l'époque des privilèges (1807-1864), ou " Rock and France " sur l'exploration d'un nouveau type de spectacles à partir des annonces publiées dans la presse spécialisée.

Pour conclure, Dezède rend plus concrète l'initiation à la recherche dont il permet d'aborder l'ensemble du processus. Le plus souvent, les travaux pratiques sont suivis de la production d'un écrit consistant à présenter le dossier auquel l'étudiant a participé ou à exploiter l'ensemble documentaire pour produire un texte musicologique, à l'image de celui consacré à la création de Siegfried à Rouen en 1900 par Charlotte Fellous (2017) ou à celle du Ring à Rouen en 1927 par Maxime Maugé-Renaünt (2018). Du point de vue de l'enseignement, cette approche offre une alternative aux cours magistraux en donnant aux étudiants la possibilité d'être acteurs de leur formation. En outre, ils acquièrent des compétences qui dépassent largement le seul cadre de la discipline à travers la gestion d'une base de données. 


\section{Conclusion}

Après sept années de développement porté par les institutions publiques avec le soutien de quelques partenaires, Dezède est devenu une référence dans l'espace des humanités numériques. Structuré sur des pratiques de la recherche historique dans le domaine des spectacles, le programme est unique dans le monde, en proposant un algorithme capable de générer des chronologies événementielles mettant en relation des données complexes. Les chercheurs s'en emparent petit à petit, pour verser leurs corpus et contribuer à rendre visible une partie de l'histoire des spectacles. Des efforts sont encore à produire pour que cet outil puisse devenir parfaitement autonome, en moyens humains et en moyens techniques. Pour parvenir à cette autonomie, il est prévu de développer des formulaires étape par étape pour guider les contributeurs dans l'utilisation des différents modèles, sur la base de quelques scénarios simples - comment créer une source ? comment la lier à un événement ? comment créer une œuvre ? etc. -, auxquels sera associé un ensemble de tutoriels d'accompagnement. Pour faciliter l'exploitation et l'analyse des données par les utilisateurs, plusieurs nouvelles fonctionnalités sont envisagées comme la généralisation de l'outil de transcription, la génération automatique de statistiques, l'indexation automatique des dossiers, un incipitaire, etc. Parmi elles, une bibliothèque numérique des spectacles est actuellement en cours d'implémentation, pour permettre une interrogation croisée des imprimés et manuscrits consacrés à l'histoire des spectacles produits depuis la fin de l'Ancien Régime. Le projet CORNUM, à l'origine de ce développement, invite également à renforcer l'interopérabilité des données, en adoptant les standards du Web sémantique, notamment Dublin Core pour intégrer Isidore, et plus généralement pour donner plus de visibilité aux données dans les grandes bases de données internationales. Ainsi, $D e$ zède est un projet numérique vivant et partagé, que ce soit du point de vue des contenus alimentés chaque jour par les utilisateurs ou de celui des améliorations techniques. Son actualité est en effet relayée auprès de la communauté scientifique et civile par un carnet de recherche hébergé sur la plateforme Hypothèses (Carnet Dezède, https://dezede.hypotheses.org) et une page Facebook consacrée au projet (https://www.facebook.com/dezede.org). Ces outils de communication visent précisément à sortir de l'isolement académique pour rendre visibles les contenus auprès des publics.

\section{Bibliographie}

Association française des orchestres. 2014. "Association française des orchestres ». Dezède. 23 octobre 2014. https://dezede.org/dossiers/id/38/.

Bellaigue, Camille. 1888. L’Année musicale : octobre 1886 à octobre 1887(-1893). 7 vol. Paris : C. Delagrave. http://gallica.bnf.fr/ark:/12148/cb34511412b/date.

Bernard, Élisabeth. 1976. Le Concert symphonique à Paris entre 1861 et 1914 : Pasdeloup, Colonne, Lamoureux. Thèse de doctorat. Paris : université Paris 1.

Bernard, Élisabeth. 1980. Programmes de la Société des concerts du Conservatoire de 1828 à 1870. 2 vol. Paris : E. Bernard. 
BNF s.d. " ISNI [International Standard Name Identifier] ». BNF. https://www.bnf.fr/fr/isni-international-standard-name-identifier.

Bouteiller, Jules-Édouard. 186o. Histoire complète et méthodique des théâtres de Rouen. 4 vol. Rouen : Giroux et Renaux ; Métérie.

Branger, Jean-Christophe. 2016. "Opéra Théâtre de Metz (1919-1939) ". Dezède. $1^{\mathrm{er}}$ décembre 2016. https://dezede.org/dossiers/id/234/ (dossier en cours de réalisation).

Brunel, Lucille. 2015. " Le séjour de Cornélie Falcon à Rouen (avril 1837) ". Dezède. 23 octobre 2015. https://dezede.org/dossiers/id/47/.

Carrère-Saucède, Christine. 2014. « Labiche en province (1837-1888) ». Dezède. 2 avril 2014. https://dezede.org/dossiers/id/45/.

Comettant, Oscar. 1894. La Musique de chambre, années 1893-1898. Séances musicales données dans les salons de la maison Pleyel, Wolf et $C^{i e}$, reproduction des programmes et comptes rendus, pour servir à l'histoire de la musique contemporaine. 6 vol. Paris : impr. de Gautherin.

Detcheverry, Arnaud. 1860. Histoire des théâtres de Bordeaux, depuis leur origine dans cette ville jusqu'à nos jours. Bordeaux : J. Delmas. https://gallica.bnf.fr/ark :/12148/bpt6k5742923h.

Élart, Joann. 2004. Catalogue des fonds musicaux conservés en Haute-Normandie. Tome I : bibliothèque municipale de Rouen. Volume 1 : fonds du Théâtre des Arts (XVIII et XIX ${ }^{e}$ siècles). Patrimoine musical régional. Rouen : Publications de l'université de Rouen.

Élart, Joann. 2013a. « Dezède, un portail pour écrire l'histoire du Rock : l'exemple des concerts rock en Haute-Normandie de 1968 à 1977 et l'arrivée du Punk ». Communication au colloque international This Is The Modern World. Pour une histoire sociale du rock, organisé par Florence Tamagne et Arnaud Baubérot, université Charles-de-Gaulle Lille 3, 1315 juin 2013.

Élart, Joann. 2013b. « La création française de Samson et Dalila à Rouen en 1890 ». Dezède. 15 février 2013. https://dezede.org/dossiers/id/1/.

Élart, Joann. 2014a. "Dezède : portail pour la valorisation de l'histoire de l'Opéra-Comique ». Conférence de présentation de délégation CNRS 2014-2015, université Paul-Valéry-Montpellier 3, Institut de recherche sur la Renaissance, l'âge classique et les Lumières, 9 octobre 2014.

Élart, Joann. 2014b. "L'Opéra-Comique sous la Restauration (1814-1830) ». Dezède. https://dezede.org/dossiers/id/54/ (dossier en cours de réalisation).

Élart, Joann. 2014c. " Boieldieu en France ". Dezède. https://dezede.org/dossiers/id/59/ (dossier en cours de réalisation).

Élart, Joann. 2016a. «Dezède, une application Web pour l'archivage des spectacles et de leurs sources ». Communication au Séminaire pluridisciplinaire d'histoire culturelle 20152016 organisé par Stéphanie Loncle, IMEC, abbaye d'Ardenne, 21 janvier 2016.

Élart, Joann. 2016b. « Dezède intègre le Consortium Musica ! C Carnet Dezède. 25 mars 2016. https://dezede.hypotheses.org/231.

Élart, Joann. 2016c. « De l’internationalisation de Dezède ». Carnet Dezède. 13 octobre 2016. https://dezede.hypotheses.org/186.

Élart, Joann. 2016d. « De l'initiation à la transcription de sources dans Dezède ». Carnet Dezède. 29 février 2016. https://dezede.hypotheses.org/166.

Élart, Joann. 2017a. « Rock and France ». Dezède. https://dezede.org/dossiers/id/261/.

Élart, Joann. 2017b. " Une histoire complète et méthodique des théâtres de Rouen publiée par le docteur Jules-Édouard Bouteiller entre 1860 et 1880 ». Territoires contemporains 8 (Séverine Féron et Patrick Taïeb, «Écrire l'histoire du théâtre. L'historiographie des institutions lyriques françaises, 1780-1914 »). http://tristan.u-bourgogne.fr/CGC/publications/historiographie_institutions_lyriques_francaises/Joann_Elart.html.

Élart, Joann. 2019. " Hommages à Pierre Corneille à Rouen d'un centenaire à l'autre (17841884)». Dezède. https://dezede.org/dossiers/id/68/.

Élart, Joann, Hervé Lacombe, Olivier Morand et Patrick Taïeb. 2005. « Répertoire des programmes de concert en France : mode d'emploi ».

Élart, Joann et Yannick Simon. 2013a. « Une nouvelle solution numérique pour écrire l'histoire des spectacles : Dezède ». Communication au Francophone Music Criticism Network Meeting 2013, Bibliothèque historique de la ville de Paris, 2 juillet 2013. 
Élart, Joann et Yannick Simon. 2013b. "Dezède et les éphémères ". Communication aux Journées professionnelles 2013 de l'Association internationale des bibliothèques musicales (AIBM), Cité de la musique, 9 décembre 2013.

Élart, Joann et Yannick Simon. 2013c. "Concerts à l'abbaye de Royaumont (1936-1977)». Dezède. https://dezede.org/dossiers/id/24/.

Élart, Joann, Yannick Simon et Patrick Taïeb. 2012. «Dezède ». BNF, catalogue général. http ://catalogue.bnf.fr/ark:/12148/cb43696753r.

Élart, Joann, Yannick Simon, Patrick Taïeb et Bertrand Bordage. 2013. «Inauguration de la base Dezède, un outil au service de la chronologie des spectacles ». Communication au colloque Les Emplois dans l'art lyrique français, organisé par Patrick Taïeb, l'Opéra-Comique et le Palazzetto Bru Zane - Centre de musique romantique française, Opéra-Comique, 2021 février 2013.

Élart, Joann, Yannick Simon, Patrick Taïeb et Bertrand Bordage, avec la participation de Christine Carrère-Saucède, Thomas Vernet et Denis Tchorek. 2014. " Le répertoire et la base Dezède ». Session au colloque Le Répertoire en question, organisé par Patrick Taïeb, Opéra-Comique, 20-21 mai 2014.

Elwart, Antoine. 1860. Histoire de la Société des concerts du Conservatoire impérial de musique, avec dessins, musique, plans, portraits, notices biographiques, etc. Paris : S. Castel. http ://gallica.bnf.fr/ark:/12148/bpt6k6480144w.

Elwart, Antoine. 1864a. Histoire de la Société des concerts du Conservatoire impérial de musique, avec dessins, musique, plans, portraits, notices biographiques, etc. $2^{\mathrm{e}}$ éd. Paris : S. Castel. http://gallica.bnf.fr/ark:/12148/bpt6k96167655.

Elwart, Antoine. 1864b. Histoire des concerts populaires de musique classique, contenant les programmes annotés de tous les concerts donnés au Cirque Napoléon depuis leur fondation jusqu'à ce jour, suivie de six esquisses sur la vie et les œuvres de J. Haydn, Mozart, Beethoven, Weber, Mendelssohn et R. Schumann. Paris : S. Castel. http://gallica.bnf.fr/ark:/12148/bpt6k648 01459.

Fellous, Charlotte. 2017. «La création de Siegfried à Rouen en 1900 ». Carnet Dezède. 13 juin 2017. https://dezede.hypotheses.org/506.

Fétis, François-Joseph. 1837. Résumé philosophique de l'histoire de la musique. Biographie universelle des musiciens. 3, CA-DZ. Bruxelles, Paris : Méline Cans et $\mathrm{C}^{\mathrm{ie}}$, Firmin Didot.

Geispitz, Henri. 1913. Histoire du Théâtre des Arts de Rouen. 1882-1913 (d'après le manuscrit de Charles Vauclin). Vol. 1. Rouen, France : Lestringuant. https://bibliotheques-specialisees.paris.fr/ark :/73873/pf0000353810.

Geispitz, Henri. 1951. Histoire du Théâtre des Arts de Rouen. 1915-1940. Vol. 2. Rouen, France : Imp. Lecerf.

Gilles, Adolphe. 1908. «Collection Gilles ». Archives municipales, Montpellier.

Jardin, Étienne. 2012. «Sédu* dans le Théâtre-Italien ». Transposition. Musique et sciences sociales 2 (mai). https://doi.org/10.4000/transposition.114.

Lefebvre, Léon. 1901. Histoire du théâtre de Lille, de ses origines à nos jours. 6 vol. Lille, France : Lefebvre-Ducrocq.

Maugé-Renaünt, Maxime. 2018. « Le Ring à Rouen en 1927 ». Carnet Dezède. 16 mars 2018. h ttps://dezede.hypotheses.org/917.

Mongrédien, Jean. 2008. Le Théâtre-Italien de Paris (1801-1831) : chronologie et documents. 8 vol. Perpetuum mobile. Lyon/Venise : Symétrie/Palazzetto Bru Zane.

Moreau, Léandre. 1872. Histoire du théâtre à Marseille : le Grand-théâtre (1792-1793). Marseille : impr. de T. Samat. http://gallica.bnf.fr/ark:/12148/bpt6k58220196.

Morvannou, Claire. 2014. "La Traviata en France (1861-1901) ». Dezède. https://dezede.org/dossiers/id/76/ (dossier en cours de réalisation).

Opéra-Comique. 2014. "Archives de l'Opéra-Comique ».Dezède. 13 novembre 2014. https:// dezede.org/dossiers/archives-opera-comique/.

Pierre, Constant. 1975. Histoire du Concert spirituel (1725-179o). Publications de la Société française de musicologie 3. Paris : Société française de musicologie.

Pierre, Constant. 200o. Histoire du Concert spirituel (1725-1790). Publications de la Société française de musicologie $3.2^{\mathrm{e}}$ éd. Paris : Société française de musicologie.

Simon, Yannick. 2012. "Lohengrin à Rouen (1891)». Dezède. 21 novembre 2012. https://dezede.org/dossiers/id/4/. 
Simon, Yannick. 2013. "Théâtre des Arts de Rouen (1882-1940) ». Dezède. https://dezede.org/dossiers/id/7/.

Simon, Yannick. 2014. " Les voyages de Monsieur Pasdeloup (1864-1883) ». Dezède. 14 mai 2014. https://dezede.org/dossiers/id/46/.

Simon, Yannick. 2015. « Concerts Lamoureux (1881-1899) ». Dezède. 10 décembre 2015. http s://dezede.org/dossiers/id/215/.

Simon, Yannick. 2017a. "Concerts Pasdeloup (1861-1887) ». Dezède. 11 février 2017. https://d ezede.org/dossiers/id/249/.

Simon, Yannick. 2017b. "Dezède and ephemera ». Communication à l'atelier Music Performance Ephemera, Sächsische Landesbibliothek - Staats- und Universitätsbibliothek Dresden, 23-24 octobre 2017.

Taïeb, Patrick, éd. 2007. "Actes du séminaire "Histoire du concert jusqu'à la Première Guerre mondiale” (Institut universitaire de France, 2001-2005) ». Revue de musicologie 93 (1) : 164 .

Taïeb, Patrick, Hervé Lacombe, Joann Élart, Yannick Simon, Étienne Jardin, Jean-Christophe Michel et William Weber. 2009. "Le Répertoire des programmes de concerts en France ». Bulletin du groupe français de l'Association internationale des bibliothèques, archives et centres de documentation musicaux 17 (décembre) : 41-91.

Toyon, Paul-Marie de. 1865. La Musique en 1864. Documents relatifs à l'art musical recueillis et mis en ordre. Paris : A. de Vresse.

Toyon, Paul-Marie de. 1866. La Musique en 1864. Documents relatifs à l'art musical recueillis et mis en ordre [...], $2^{e}$ année. Paris : A. de Vresse. http://gallica.bnf.fr/ark:/12148/bpt6k96123058.

Vauclin, Charles. 1912. "Histoire du Théâtre-des-Arts [...]. Répertoire alphabétique et date de chacune des représentations de toutes les œuvres lyriques jouées au Théâtre-des-Arts, du commencement du XIX ${ }^{\mathrm{e}}$ siècle au 14 juillet $1912 »$. Bibliothèque municipale, Rouen.

Vernet, Thomas. 2014. "Les concerts dans les résidences royales sous la monarchie de Juillet ». Dezède (dossier en cours de réalisation).

Vesque, Charles-Théodore. 1875. Histoire des théâtres du Havre, 1717 à 1872, comprenant l'historique des anciennes et des nouvelles salles de spectacle de cette ville, le répertoire des pièces jouées jusqu'à ce jour, les représentations par les célébrités dramatiques et lyriques, les débuts et incidents s'y rattachant, épisodes, anecdotes, biographies, etc. Le Havre : impr. de Labottière \& $\mathrm{C}^{\mathrm{ie}}$ et impr. de J. Brenier \& $\mathrm{C}^{\mathrm{ie}}$.

\section{Notes}

1 Lors de la journée d'étude Rendez-vous au Théâtre des Arts de Rouen! organisée à l'opéra de Rouen Haute-Normandie le 23 février 2012 par Joann Élart et Yannick Simon.

2 Nous soulignons en italique le nom du site, s'agissant d'une publication en ligne « à mise à jour quotidienne ".

3 C'est le cas entre autres du théâtre des Champs-Élysées qui propose un portail de consultation et d'interrogation de ses archives : « Les archives. 100 ans d'histoire à votre disposition " (http://www.tce-archives.fr/) ou du projet des registres de la Comédie-Française qui met à disposition des outils d'analyse de son répertoire entre 1680 et 1793 (https:/ /www.cfregisters.org/fr/).

4 Cette étude est poursuivie plus tard par Geispitz (1913, 1951). Voir Élart (2017b).

5 La réédition de cet ouvrage en 1864 a été l'occasion d'y ajouter les programmes des concerts des années 1860 à 1863 (Elwart 1864a).

6 Cet ensemble de programmes est désormais inséré dans le répertoire exhaustif des Concerts Pasdeloup entre 1861 et le décès de leur fondateur en 1887. Voir Simon (2017a); s'agissant des références au contenu de Dezède, nous avons fait le choix de ne pas mentionner la date de dernière consultation qui est, dans tous les cas, identique à celle de la parution du présent article.

7 Les quatre volumes proposent un historique des associations, les programmes, une analyse des programmes, un index des compositeurs et des interprètes. 
8 On doit cette mise en forme à Antoine Bloch-Michel, archiviste-paléographe, bibliothécaire au département de la musique à la Bibliothèque nationale de France, qui a complété et indexé les quatre-vingt-cinq pages du manuscrit original de l'auteur réalisé en 1899 pour le prix Bordin de l'Institut de France et déposé à la Bibliothèque nationale en 1966. C'est lui par exemple qui rétablit les sources dans chaque programme. Voir la préface de François Lesure dans Pierre (2000, 3-4).

9 La publication de cet ouvrage a été associée à la création d'un site informatique désormais inaccessible. Il proposait en particulier un moteur de recherche qui permettait une entrée thématique dans l'ensemble des volumes et d'interroger de manière systématique des notions impossibles à isoler dans la version papier. C'est à partir de cet outil numérique qu'Étienne Jardin a par exemple produit son étude sur les "séductions musicales " (Jardin 2012).

10 Ces pratiques ont donné lieu notamment à la publication de plusieurs dizaines de catalogues dans la collection « Patrimoine musical régional ».

11 Sur le RPCF, voir Taïeb et al. (2009), Taïeb (2007).

12 Rendez-vous au Théâtre des Arts de Rouen !, journée d'étude organisée par Joann Élart et Yannick Simon à l'opéra de Rouen Haute-Normandie le 23 février 2012.

13 Comme l'adresse pérenne l'indique, il s'agit donc du premier événement créé dans $D e-$ zède, https://dezede.org/evenements/id/1/.

14 Quand la politique s'invite au spectacle, journée d'étude organisée par Joann Élart, Yannick Simon et Éric Saunier à l'université du Havre le 21 novembre 2012.

15 «Les humanités numériques et les projets de corpus en histoire du théâtre » et "Les humanités numériques et la politique nationale ", deux tables rondes organisées dans la journée Expérimentations pratiques : revoir et repenser le corpus en contexte numérique par Sara Harvey et Agathe Sanjuan, dans le cadre du colloque Remettre en jeu le passé. Métamorphoses du corpus des Registres de la Comédie-Française (1680-1793) / (2013-2016), Comédie-Française, 14-16 décembre 2015.

16 Voir Élart (2016b).

17 Joann Élart (MCF université de Rouen Normandie, GRHIS), Yannick Simon (PU université de Rouen Normandie, CÉRÉdI) et Patrick Taïeb (PU Paul-Valéry-Montpellier 3, IRCL).

18 Pour en savoir plus, voir Élart (2016c).

19 Les deux polices de caractères utilisées dans Dezède sont : avec empattements, Linux Libertine - utilisée notamment pour le logo de Wikipédia - ; sans serif, Linux Biolinum. Ces deux polices conçues par le typographe allemand Philipp H. Poll sont à source ouverte.

20 Auguste et Théodore, ou Les Deux Pages est une comédie mêlée de chants en deux actes sur un livret d'Ernest de Manteuffel et une musique de Nicolas Dezède représentée pour la première fois le 6 mars 1789 à la Comédie-Française.

21 Un premier corpus d'autorités a été constitué à partir d'une importation automatique des tables d'auteurs et d'œuvres publiées dans Élart (2004).

22 Le nombre d'événements est précisément de 44673 au 10 avril 2019.

23 Chiffres au 10 avril 2019. La publication des données dans Dezède peut être différée par l'utilisateur qui les produit, pour une raison de confidentialité. Chaque modèle de données est ainsi doté d'un « état » qui gère cette fonctionnalité.

24 Pour plus d'informations, voir l'onglet " Origines » sur la page « Bibliographie » $:$ https: //dezede.org/bibliographie.

25 Voir l'onglet « Références bibliographiques » sur la page "Bibliographie » : https://dezede.org/bibliographie.

26 Voir le cas plus intéressant de la chanteuse Maria Malibran : https://dezede.org/individus/id/2057/.

27 Plusieurs aménagements sont prévus avant la fin de l'année 2019 pour rendre différentes parties du site interopérables, notamment avec Isidore (dossiers, bibliothèque, individus, œuvres, ensembles).

28 Pour en savoir plus, voir Élart (2016d). 


\section{Auteurs}

\section{Joann Élart}

EA 3831 Grhis, université de Rouen-Normandie, Mont-Saint-Aignan, France

Joann Élart travaille sur la vie musicale en France entre la fin de l'Ancien Régime et la Restauration. Il publie le Catalogue du fonds du Théâtre des Arts de Rouen 1750-19oo (PURH, 2004), fonde avec Yannick Simon et Patrick Taïeb le portail numérique Dezède sur l'archivage et la chronologie des spectacles (https://dezede.org, 2012-), anime avec Sophie Victorien le numéro Musique et justice (Criminocorpus, 2013), codirige avec Étienne Jardin et Patrick Taïeb Quatre siècles d'édition musicale, mélanges offerts à Jean Gribenski (Peter Lang, 2014) et publie avec Pascal Dupuy les actes du colloque Rock et violences en Europe (Criminocorpus, 2018).

joann.elart@univ-rouen.fr

\section{Yannick Simon}

EA 3229 Cérédi, université de Rouen-Normandie, Mont-Saint-Aignan, France

Professeur à l'université de Rouen, Yannick Simon travaille sur la vie musicale en France sous la III ${ }^{\mathrm{e}}$ République et sous l'Occupation. Il est l'auteur de La Sacem et les droits des auteurs et compositeurs juifs sous l'Occupation (La Documentation française, 200o) et de Composer sous Vichy (Symétrie, 2009). Il a dirigé, avec Myriam Chimènes, la publication de $L a$ Musique à Paris sous l'Occupation (Fayard, 2013). Il effectue parallèlement des recherches sur la diffusion de la musique symphonique, de la musique de chambre et de l'opéra dans les régions françaises. Il a consacré deux ouvrages aux concerts populaires : L’Association artistique d'Angers (Société française de musicologie, 2006) et Jules Pasdeloup et les origines $d u$ concert populaire (Symétrie, 2011). Il est aussi l'auteur de Lohengrin : un tour de France, 1887-1891 (Presses universitaires de Rennes, 2015). yannick.simon@univ-rouen.fr

\section{Droits d'auteur}

Les contenus de la revue Humanités numériques sont mis à disposition selon les termes de la Licence Creative Commons Attribution 4.0 International. 